\title{
Artificial Intelligence for Diabetes Management and Decision Support: Literature Review
}

Ivan Contreras ${ }^{1}, \mathrm{PhD}$; Josep Vehi ${ }^{1,2}, \mathrm{PhD}$

\author{
Corresponding Author: \\ Josep Vehi, PhD \\ Modeling, Identification and Control Laboratory \\ Institut d'Informatica i Aplicacions \\ Universitat de Girona \\ Campus Montilivi. Edifici P4 \\ Girona, 17003 \\ Spain \\ Phone: 34620131826 \\ Email: josep.vehi@udg.edu
}

${ }^{1}$ Modeling, Identification and Control Laboratory, Institut d'Informatica i Aplicacions, Universitat de Girona, Girona, Spain

${ }^{2}$ Centro de Investigación Biomédica en Red de Diabetes y Enfermadades Metabólicas Asociadas, Girona, Spain

\begin{abstract}
Background: Artificial intelligence methods in combination with the latest technologies, including medical devices, mobile computing, and sensor technologies, have the potential to enable the creation and delivery of better management services to deal with chronic diseases. One of the most lethal and prevalent chronic diseases is diabetes mellitus, which is characterized by dysfunction of glucose homeostasis.

Objective: The objective of this paper is to review recent efforts to use artificial intelligence techniques to assist in the management of diabetes, along with the associated challenges.

Methods: A review of the literature was conducted using PubMed and related bibliographic resources. Analyses of the literature from 2010 to 2018 yielded 1849 pertinent articles, of which we selected 141 for detailed review.

Results: We propose a functional taxonomy for diabetes management and artificial intelligence. Additionally, a detailed analysis of each subject category was performed using related key outcomes. This approach revealed that the experiments and studies reviewed yielded encouraging results.

Conclusions: We obtained evidence of an acceleration of research activity aimed at developing artificial intelligence-powered tools for prediction and prevention of complications associated with diabetes. Our results indicate that artificial intelligence methods are being progressively established as suitable for use in clinical daily practice, as well as for the self-management of diabetes. Consequently, these methods provide powerful tools for improving patients' quality of life.
\end{abstract}

(J Med Internet Res 2018;20(5):e10775) doi: 10.2196/10775

\section{KEYWORDS}

diabetes management; artificial intelligence; machine learning; mobile computing; blood glucose

\section{Introduction}

\section{Overview}

Diabetes mellitus refers collectively to a group of diseases resulting from dysfunction of the glucoregulatory system [1]. Hyperglycemia, the hallmark of diabetes, is the primary consequence of this dysregulation. Chronic hyperglycemia in diabetes is associated with long-term complications involving tissue damage and organ failure, which can decrease life expectancy and even cause death. The International Diabetes
Federation estimates that, by 2017, diabetes affected 425 million people worldwide, of whom, 4 million died in the same year. These figures are expected to increase dramatically in the coming decades, placing a rising burden on health care systems [2].

Most diabetes can be categorized into 3 subgroups: type 1 diabetes (T1D), type 2 diabetes (T2D), and gestational diabetes (GDM). Over the long term, T2D patients become resistant to the normal effects of insulin and gradually lose their capacity to produce enough of this hormone. A wide range of therapeutic 
options are available for patients with T2D. At the early stages of disease, they commonly receive medications that improve insulin secretion or insulin absorption, but eventually they must receive external doses of insulin. On the other hand, T1D patients have severe impairments in insulin production, and must use external insulin exclusively to manage their blood glucose (BG). Treatment of T1D requires consistent doses of insulin through multiple daily injections (MDIs) or continuous subcutaneous insulin infusion (CSII) using a pump. GDM is treated similarly to T2D, but only occurs during pregnancy due to the interaction between insulin and hormones released by the placenta.

In each class of diabetes, timely diagnosis, education of patients in self-management, and continuous medical care are required to prevent acute complications (eg, ketoacidosis) and minimize the risk of long-term complications (eg, nephropathy, retinopathy, diabetic foot, cardiovascular disease, or stroke). In addition to medication, management of diabetes requires adherence to an array of self-care behaviors that are often very burdensome for patients: carefully scheduling meals, counting carbohydrates, exercising, monitoring BG levels, and adjusting endeavors on a daily basis. The effects of nonadherence to recommended treatment are not immediately evident and long-term complications may take years to develop. Accordingly, diabetes therapy is complex, and therapeutic decisions need to take into account diverse medical factors and lifestyle-related activities that must be optimized to improve diabetic patients' quality of life.

Artificial intelligence (AI) is a quickly growing field, and its applications to diabetes research are growing even more rapidly as shown in Figure 1, which is a gross estimate of the number of related articles in the Google Scholar database.
In the literature, intelligent algorithms are widely used in data driven methods to support advanced analysis and provide individualized medical aid. There is also evidence that an increasing number of health care companies are applying these techniques [3]. Short-term prospects indicate they are likely to have considerable success in clinical practice. The main reasons for this growth include the explosive increase in the amount of available information, along with the improved performance of intelligent methodologies capable of handling and processing this information, both of which have led to the development of tools and applications which can enhance the effective management of complicated diseases, including diabetes and cancer.

Over the last decade, the entire paradigm of diabetes management has been transformed due to the integration of new technologies such as continuous glucose monitoring (CGM) devices and the development of the artificial pancreas (AP), along with the exploitation of data acquired by applying these novel tools. AI is attracting increased attention in this field because the amount of data acquired electronically from patients suffering from diabetes has grown exponentially. By means of complex and refined methods, AI has been shown to provide useful management tools to deal with these incremental repositories of data. Thus, AI has played a key role in the recognition of these systems as routine therapeutic aids for patients with diabetes.

The literature offers ample evidence of the use of artificial intelligence methods in the field of diabetes, such as in general surveys [4,5] or in particular domains, for example early diagnosis [6]. In this manuscript, we describe the latest efforts and advances in the application of AI methodologies to diabetes management and decision support. Background information on AI methods is provided in the remainder of the Introduction section.

Figure 1. The number of published articles in Google Scholar that include the terms "diabetes" and "artificial intelligence.".

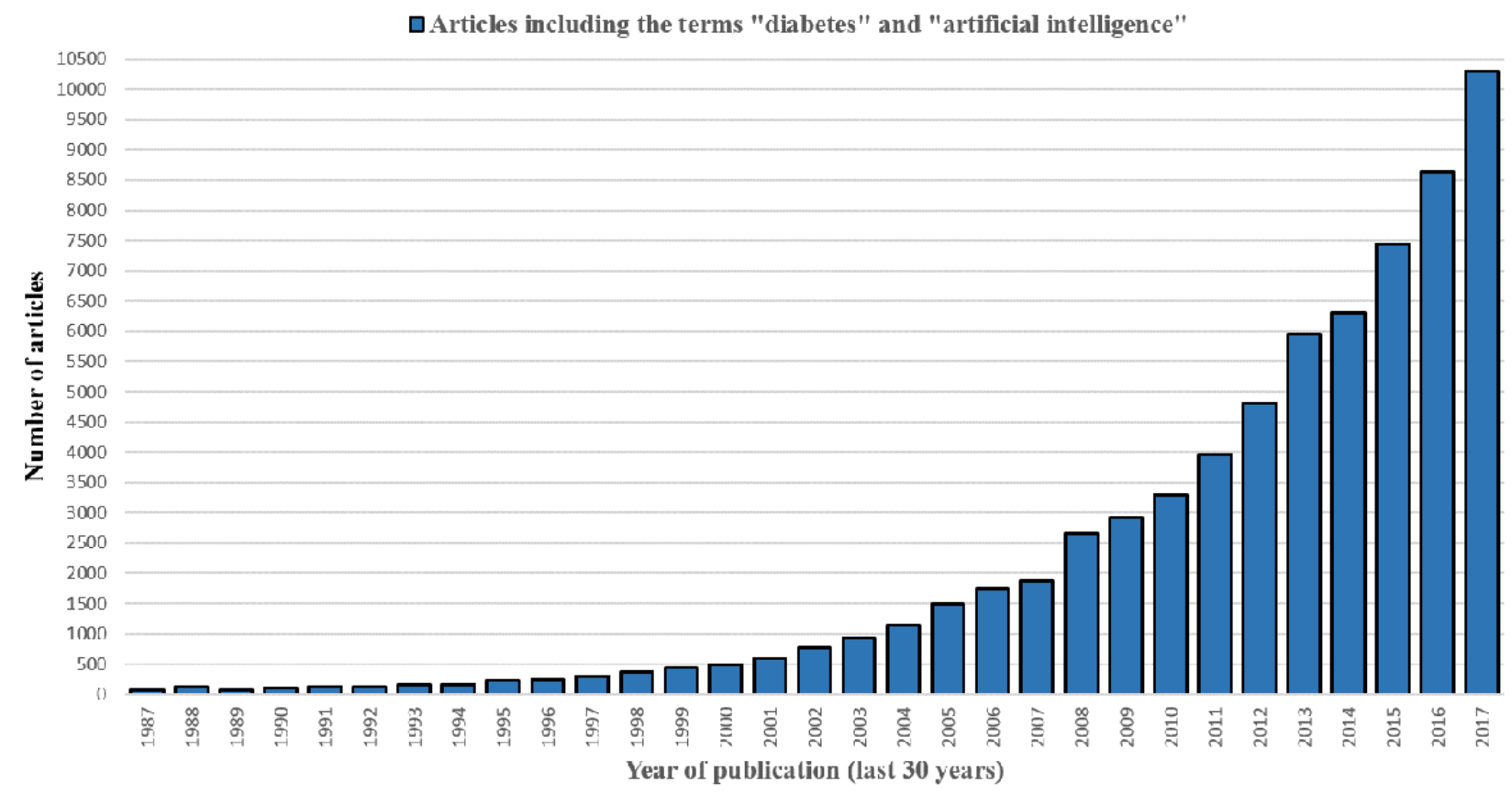


We then provide a detailed description of the methodological approach used in this review, report the results of a literature analysis, discuss studies on various sub-topics, and conclude with a brief summary and a discussion of future challenges.

\section{Artificial Intelligence Techniques}

Defining the concept of AI, computational intelligence, or machine intelligence is not a trivial undertaking. In this paper, we refer to AI as a branch of computer science that aims to create systems or methods that analyze information and allow the handling of complexity in a wide range of applications (in this case, diabetes management). Although the application of AI algorithms involves highly technical and specialized knowledge, this has not prevented AI from becoming an essential part of the technology industry and making contributions to major advances within the field. This section will provide a short overview of several well-known computational intelligence paradigms. For a more in-depth discussion of various intelligent algorithms, theoretical results, and applications, the reader is referred to the following book by Nilsson [7]. In this study, we categorized methodologies with respect to the objective sought: to explore and discover information, to learn using information, or to extract conclusions from information (see Figure 2).

\section{Learning from Knowledge}

Acquisition of knowledge is a key requirement of solutions intended to exhibit intelligent behavior. Because learning is an effective way to introduce such knowledge, most AI studies to date have employed learning techniques (see Figure 3). The primary aim of learning from knowledge is to allow computers to learn automatically without human intervention or assistance. This process could involve any method that includes some inductive component, ranging from a simple Kalman filter to a complex convolutional neural network. No method is inherently better than any other; each is more or less well-suited to different scenarios, for example, a softer learning curve, faster execution, or more flexible solutions. Furthermore, the performances of various methods are closely related to the quality and quantity of data: when more information is gathered, and less noise is present in the data, better solutions can be obtained. The most important families of techniques are artificial neural networks (ANNs), support vector machines (SVMs), random forest (RF), evolutionary algorithms (EAs), deep learning, Naïve Bayes, decision trees, and regression algorithms.

\section{Exploration and Discovery of Knowledge}

The discovery of knowledge revolves around the exploration and creation of algorithms for retrieving potential information from databases, commonly referred to as knowledge discovery in databases (KDD). The primary objective of KDD is identification of valid, potentially useful, and understandable information. KDD involves evaluation and interpretation of patterns and models for making decisions about what does and does not constitute knowledge, that is, distinguishing between data that are useful and those that are (in the context of interest) useless. Therefore, KDD requires broad and deep knowledge about the area of study.

The overall KDD process may be characterized into 6 steps in the cross industry standard process for data mining (CRISP-DM) model (Figure 4) [8]: business understanding, data understanding, data preparation, data modeling, evaluation of the model, and deployment. The application of data mining modelling is the most technical stage of the process. Techniques for data mining have taken much of their inspiration from learning algorithms and statistics, although the two types of approaches have different objectives. The most important data mining tasks involve the detection of anomalies, identification of dependencies between variables, regression, clustering, and classification. Some examples of representative techniques for this process are k-means, the k-nearest neighbor algorithm, and hierarchical clustering (HC).

Figure 2. A taxonomy of some of the best known artificial intelligence methods.

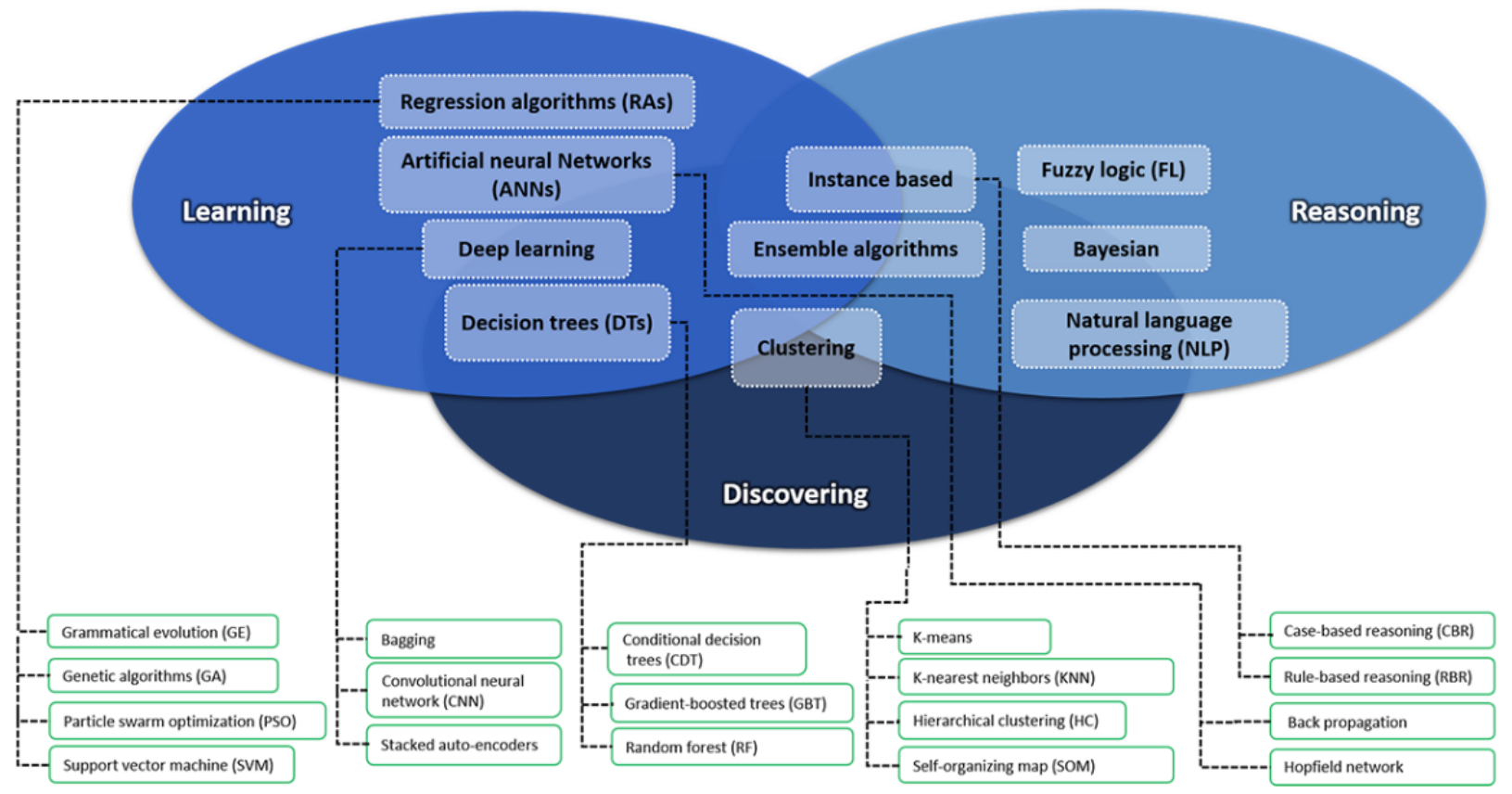


Figure 3. A general diagram of the learning algorithm process.

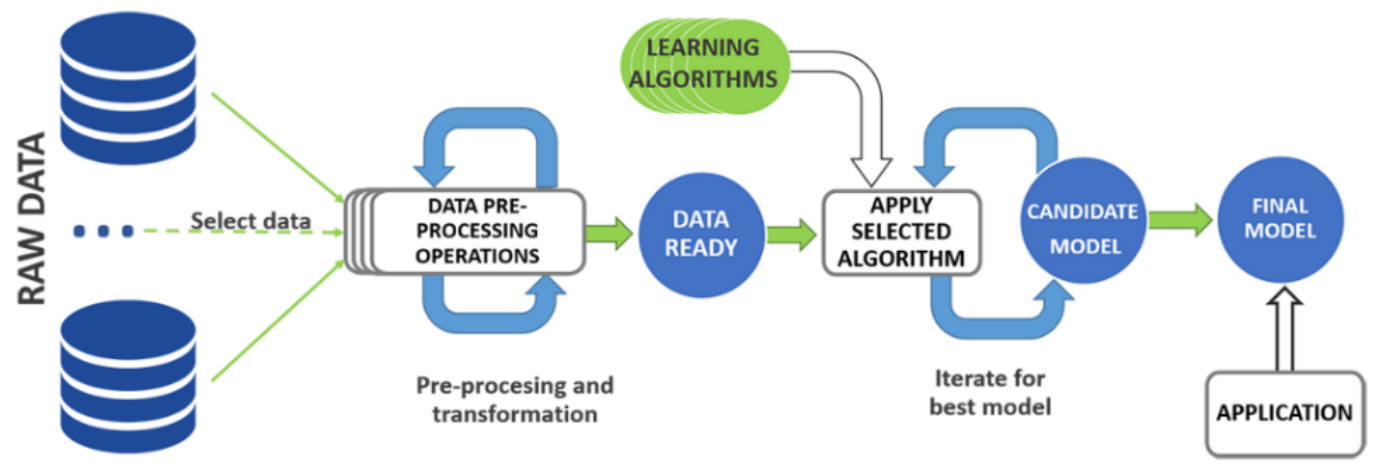

Figure 4. General CRISP-DM model for the knowledge discoveryin databases (KDD) process.

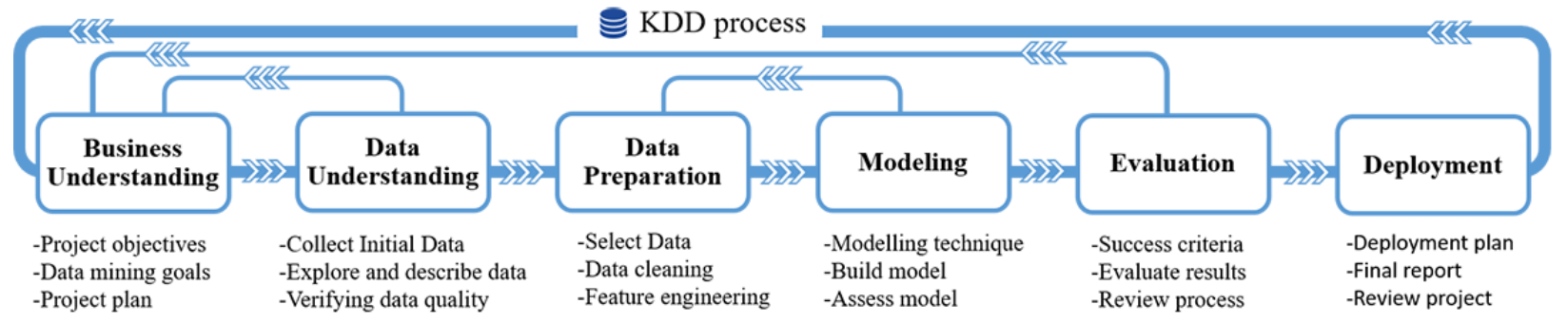

\section{Reasoning from Knowledge}

In this discourse, the idea of reasoning from knowledge denotes the creation of precise and effective ways to generate inferences in more precise and robust ways. Thus, reasoning from knowledge involves the use of logical techniques such as deduction and induction to generate conclusions from the available knowledge. The primary objective of systems that implement reasoning mechanisms is to perform tasks at a human-expert level in a narrow, specialized manner within the domain of interest. Such systems commonly apply heuristics to guide reasoning and reduce the search space of possible solutions.

These systems are based on 3 main components. First, a knowledge acquisition system is used to gather and collect inferences that can be used for further development. In this context, such a system is used to extract new rules and gather information. Second, a knowledge base, characterized by rules and information, is used for problem solving. Important aspects here include relations, conditions, recommendations, directives, and strategies. Finally, the inference engine links the knowledge base with the gathered information. Overall, this process facilitates reasoning, whereby the system becomes able to facilitate the realization of the anticipated solution. It is possible for a system structured on this basis to transfer expert knowledge directly to the knowledge base. This, in turn, helps to build new solutions based on previous cases, or to deal with ambiguous concepts and uncertainty. Representatives of these tasks include rule-based reasoning, case-based reasoning (illustrated in Figure 5), and fuzzy logic.

Figure 5. The case-based reasoning circle.

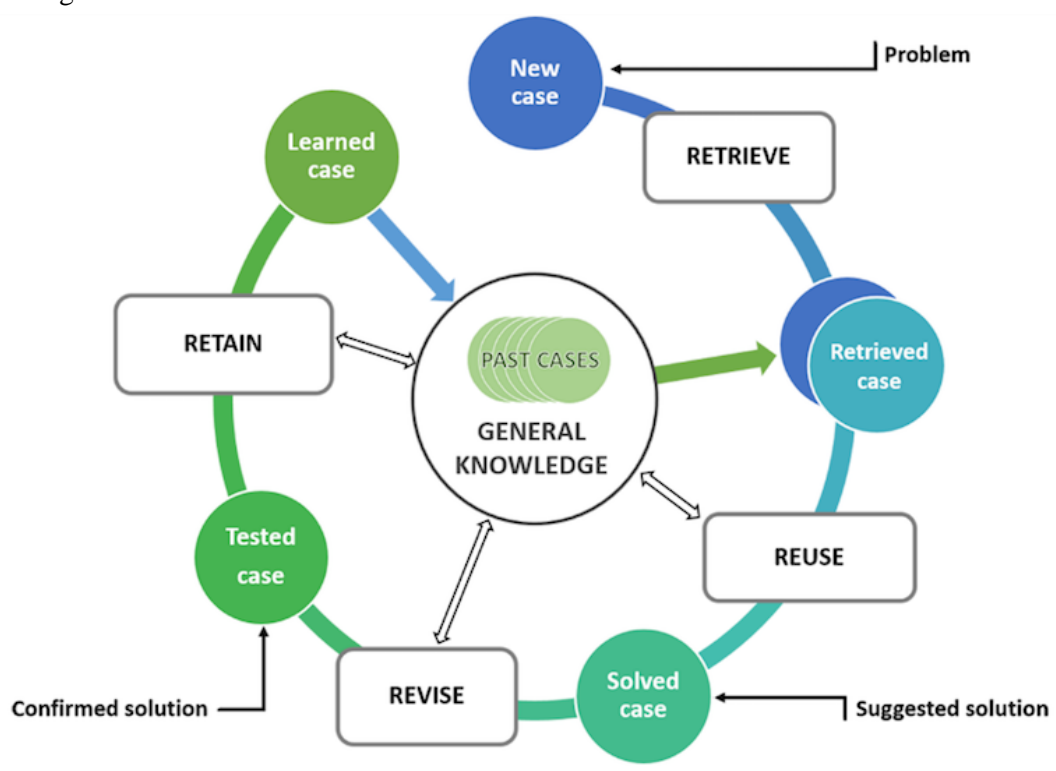




\section{Methods}

A review of the literature was conducted using the PubMed database. The selection of this bibliographic system as the primary data source was motivated by the sharp increase in the number of articles in the database and the strong link between these articles and the health care sector. PubMed has been validated as a reliable tool for retrieving information on medical research and clinical applications. Only English-language documents published between 2010 and 2018 were considered. The search terms listed in Textbox 1 were used to identify terms in the abstracts, titles, and keywords of the documents.

The search terms were explored and combined, yielding 1841 "hits." The terms "diabetes," "management," "artificial pancreas," and "blood glucose" were combined with the remaining terms using a conjunctive operator, and these terms were used as keywords to create individual datasets comprising all references to the following phrases: "artificial intelligence" (186), "computational intelligence" (179), "machine learning" (88), "data mining" (111), "deep learning" (3), "k-means" (9), "fuzzy logic" (24), "heuristic" (10), "clustering analysis" (281), "Bayes" (19), "decision tree" (67), "random forest" (21), "particle swarm optimization" (7), "pattern recognition" (31), "genetic algorithm" (43), "supervised algorithm" (14), "unsupervised algorithm" (9), "knowledge-based" (14), "case-based reasoning" (11), "decision support system" (71), "self-organizing map" (4), "evolutionary computation" (2), "neural network" (72), "natural language processing" (34), "reinforcement learning" (6), clustering (510), and "support vector machine" (23). Each of these datasets was then combined to build an objective dataset of articles. A comprehensive review was performed of all references cited in the datasets. Finally, the bibliography of the reviewed articles was thoroughly explored to find titles with relevance to the main focus of this study.

The complete method is summarized in Figure 6. The resultant final collection of articles was divided into various categories, designed to assist in the grouping of studies according to their shared and specific characteristics. Over the course of the systematic review, the subcategories were fused and fixed to accommodate the merging of information.

Textbox 1. The terms used in the search queries.

Artificial intelligence; Artificial neural network; Artificial pancreas; Blood glucose; Case-based reasoning; Cluster analysis; Clustering; Computational intelligence; Data mining; Decision support systems; Decision tree; Deep learning; Diabetes; Evolutionary computation; Fuzzy logic; Genetic algorithm; Heuristic; K-means; Knowledge-based; Machine learning; Management; Natural language processing; Naïve Bayes; Particle swarm; Pattern recognition; Random forest; Reinforcement learning; Self-organizing map; Supervised learning; Support vector machine; Unsupervised learning

Figure 6. Summary of the review process and classification of articles into a set of subdomains.

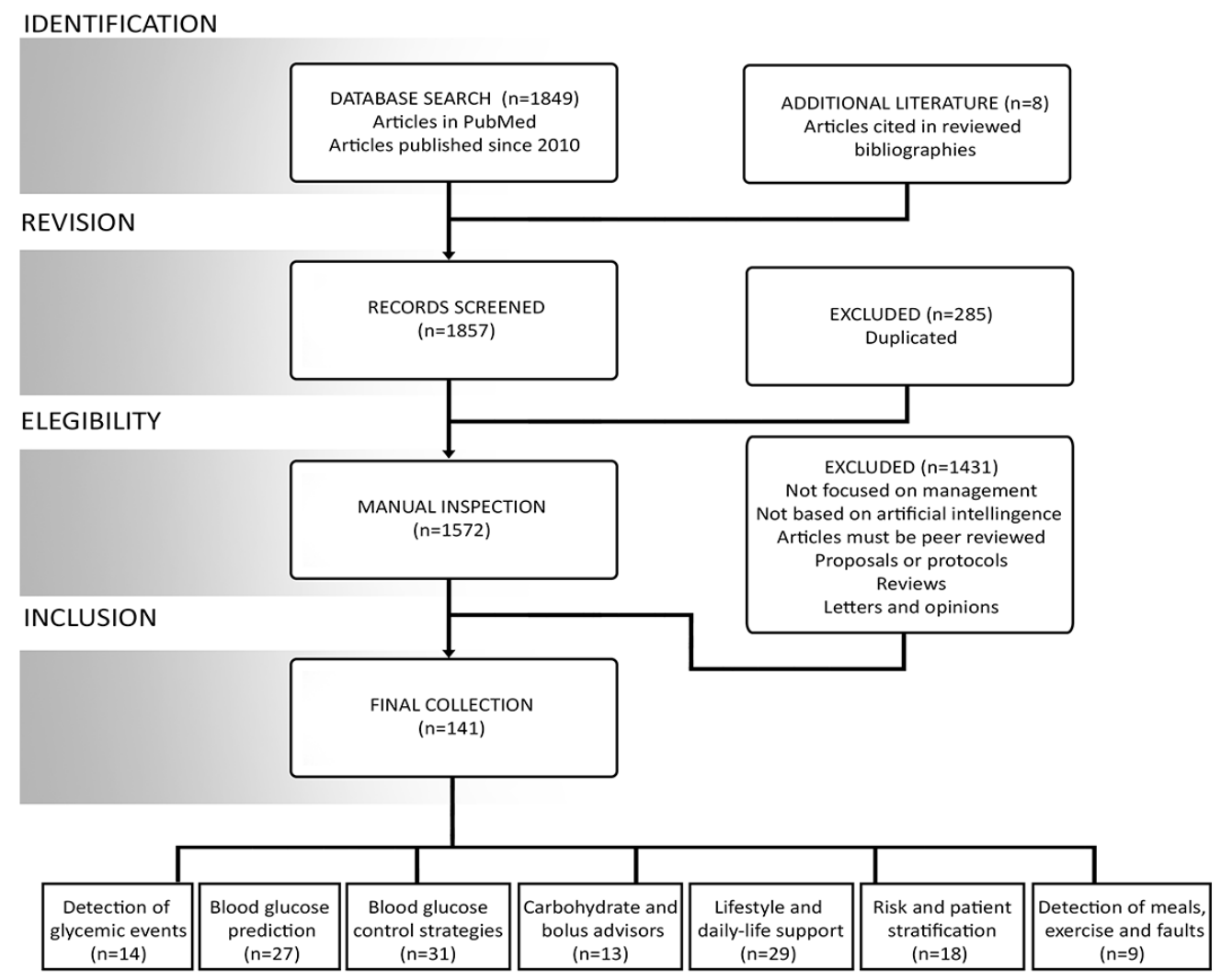




\section{Results}

\section{Main Findings}

Ultimately, 141 papers were included in the review. The potential of AI to enable diabetes solutions has been investigated in the context of multiple critical management issues. In this section, we use the following proposed diabetes management categories to summarize the latest contributions and the results described in the reviewed articles:

- Blood glucose control strategies

- Blood glucose prediction

- Detection of adverse glycemic events

- Insulin bolus calculators and advisory systems

- Risk and patient personalization

- Detection of meals, exercise and faults

- Lifestyle and daily-life support in diabetes management

As seen in Figure 7, the majority of the papers were published in the last 3 years, reflecting a clear acceleration in the application of AI techniques to diabetes management. In the following, contributions to each of the subdomains are detailed and discussed.

\section{Blood Glucose Control Strategies}

Development of the AP has been intensively pursued over the past decade. An AP consists of an automated system that mimics islet physiology, including a glucose sensor, a closed-loop control algorithm, and an insulin infusion device. The ultimate goal of an AP system is to improve overall diabetes management and reduce the frequency of life-threatening events associated with T1D. The algorithms used by the AP to calculate insulin dosage have been intensively investigated, either using data from diabetic patients or computer simulated patients, commonly named virtual patients (VP). The major candidate algorithms are derived from traditional control engineering theory; however, AI has become more established over the past few years and could ultimately provide better candidates to meet the challenges of an AP [9].
Although AI and control engineering have converged to some extent as the two fields incrementally exchange methods, here we will focus on studies dealing with closed-loop algorithms based on AI techniques. We direct interested readers to a recent comprehensive review on AP systems [10].

Three main AI methodologies have been established as control techniques in recent years: FL, ANNs, and reinforcement learning (RL). Most alternatives to control engineering algorithms are based on FL. Controllers apply FL theory to imitate the lines of reasoning of diabetes caregivers. Thus, the primary benefit of FL over classic control engineering is the ability to deal with nonlinearities and uncertainties. However fuzzy logic systems have not yet been proven to clearly outperform well-tuned classical approaches.

MD-Logic [11,12] was developed by authors who sought to individualize glycemic control using a fuzzy controller. Two feasibility studies were conducted in cohorts of 7 T1D patients to introduce the methodology and test the viability of the controller. Subsequently, a randomized crossover trial was conducted in 12 T1D patients [13]. The results suggested that the fuzzy method could improve nocturnal BG control without increasing the risk of hypoglycemia. Following the success of these feasibility studies, the authors performed a randomized crossover study of 56 young patients over 3 days [14]. The results confirmed a reduced rate of nocturnal hypoglycemia and superior glycemic control in comparison with insulin pump treatment. In a home-based randomized trial of $15 \mathrm{~T} 1 \mathrm{D}$ patients [15], the authors compared the fuzzy AP and sensor-augmented pump over 4 nights; the results confirmed the feasibility, safety, and efficiency of their approach in a home setting. Later, an extended study of 24 T1D patients during 6 weeks of nocturnal control demonstrated the safety and effectiveness of long-term use of a FL-based controller. In a recent clinical trial evaluating remote patient monitoring of the FL controller, the AP was tested in 75 T1D patients for 4 consecutive nights. The results demonstrated safe and efficient glycemic control. Further studies will evaluate the MD-Logic controller implemented in MiniMEd 690G [16].

Figure 7. Number of articles reviewed according to subdomain and year of publication (BG: blood glucose).

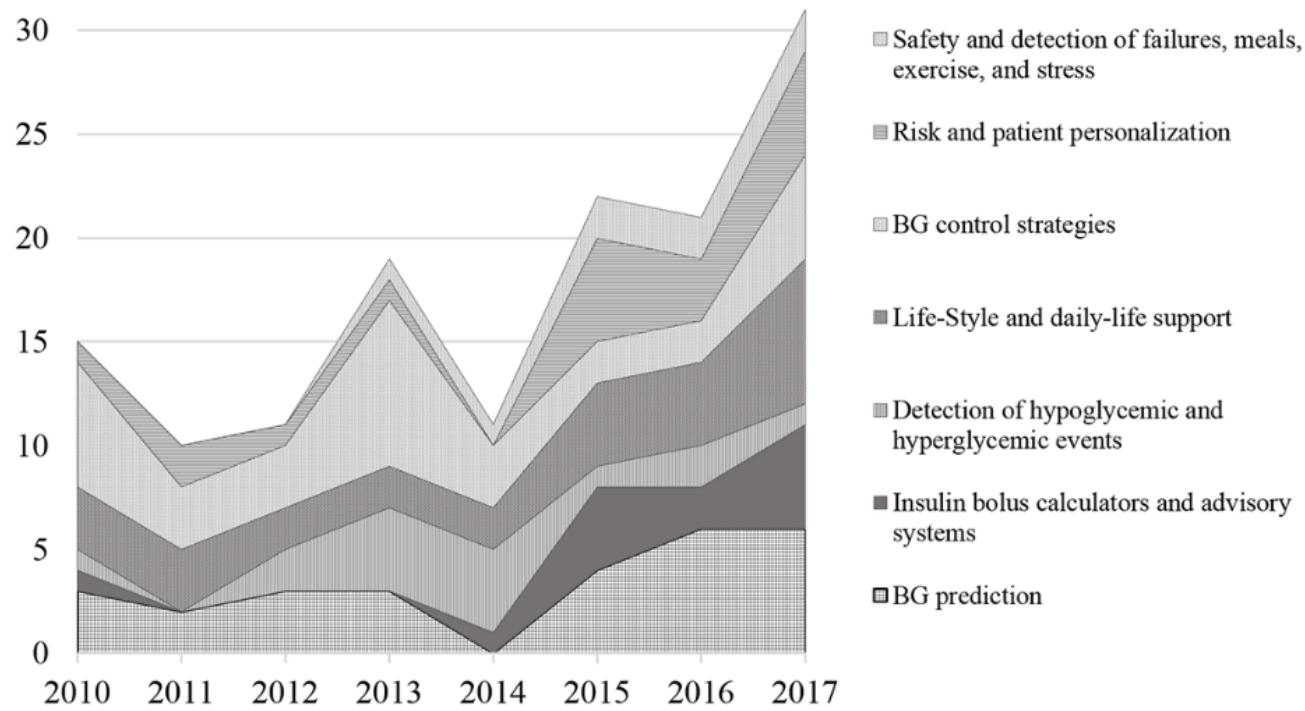


Other research groups have also investigated the application of FL to BG control. For example, Mauseth et al [17] reported a FL controller designed to personalize glycemic control. They tested it in 30 virtual patients on the UVA/Padova T1D simulator. Next, to demonstrate the feasibility of their approach, they conducted a pilot study in 12 T1D patients [18]. In a later study, they proposed stressing a fuzzy controller with high-fat meals and exercise and tested this approach in a trial with 10 T1D patients [19]. The results revealed deficits in their previous approach and ultimately led to improvements in the FL controller.

Other FL approaches have been tested using virtual patients or simulations [20-24]. For example, Miller et al reported a fuzzy controller combined with a learning algorithm that extracted initial patient profiles using open-loop data [23]. Another example was provided by Dinani et al [24], who suggested combining fuzzy and sliding-mode controllers, with the goal of using feedback to govern the insulin delivery rate more aggressively.

Another AI method that has been increasingly adopted in the area of control algorithms is the so-called reinforcement algorithm [25]. Daskalaki et al presented an adaptive, patient-specific BG control strategy based on the Actor-Critic learning approach and tested their approach on 28 virtual T1D patients (adults, adolescents, and children) [26].

Another AP study based on RL was proposed by Daskalaki et al, who proposed using an Actor-Critic algorithm to optimize insulin infusion for personalized glucose regulation, and evaluated the system using virtual patients [27]. The results revealed that their novel tuning method decreased the risk of severe hypoglycemia, especially in patients with low insulin sensitivity. Other AI-related techniques used to support the development of the AP included modeling of glucose metabolism with an SVM [28].

Over this period, several groups proposed complementary AI algorithms to support AI controllers. Fereydouneyan et al proposed assisting the controller with a genetic algorithm (GA) that optimizes the values for two inputs and one output membership function [22], with the goal of preventing fluctuations caused by derivatives in fuzzy design. Another GA was used in the work of Catalogna et al to support an ANN controller [29]. In that case, the proposed GA optimizes network topology and learning features, instead of using the trial-and-error approach commonly adopted in ANN topology determination. In line with previous studies, Khooban et al proposed a controller assisted by particle swarm optimization that optimizes the parameters of the glucose-insulin model [21]. Following the trend of combining methods to control BG with metaheuristics, Yadav et al proposed the use of a Cuckoo search algorithm to optimize the gain of the controller [20]. Optimization of AI techniques, aside from their use in conjunction with AI controllers, have been used in other studies to determine controller parameters. For example, Tang et al used GA to mine information from patients' medical histories to generate multiple customized models [30], and Greenwood et al proposed the use of particle swarm optimization to adjust the function cost of an economic predictive control model [31].
Complementary to advances in control algorithms, efforts have been made to improve models that attempt to capture glucose-insulin dynamics. Since the publication of the seminal paper in this field [32], interest has increased in applying neural networks to identification and control of nonlinear systems. Zarkogianni et al $[33,34]$ developed a recurrent neural network trained with a real-time recurrent learning algorithm that models the BG kinetics of T1D patients and predicts BG levels using information about meal intake, BG measurements, and infused insulin. González-Olvera et al investigated the use of a fuzzy neural network in an attempt to combine the best properties of ANNs and fuzzy systems [35]. Specifically, they implemented a learning system that combines input signals, infused insulin, and BG measurements with a membership initialization based on the fuzzy c-means algorithm. The following year, Alanis et al performed a more rigorous study using a recurrent neural network trained to model BG in T1D patients [36]. The approach they implemented considered glucose absorption via carbohydrates consumption and insulin infusion as inputs. These 3 neural network studies proposed a model of insulin dynamics as the first step in the design of a control scheme, and then validated the model using T1D patient data. Their results demonstrated that they were indeed able to capture BG dynamics. More recently, 2 studies presented a complete control scheme using neural networks $[29,37]$. Both studies sought to determine whether this technique could predict and control BG excursions in T1D using patient-specific models. The first approach was tested using the hyperinsulinemic-euglycemic clamp technique in 34 rats, and the second was tested using in silico data including BG measurements, administered insulin, exercise information, and ingested food. The results of both approaches demonstrated that ANNs are accurate methods for regulation of glucose levels.

\section{Blood Glucose Prediction}

The ability to anticipate BG excursions could provide early warnings regarding ineffective or poor treatments. Thus, information collected from new technologies for diabetes management, such as the CGM devices, could lead to real-time predictions of future glucose levels. Prediction of BG levels is challenging due to the number of physiological factors involved, such as delays associated with absorption of food and insulin, and the lag associated to measurements in the interstitial tissue. Errors of the CGM also increase the difficulty of predicting BG values (approximately 9\% of the mean absolute relative difference for the best sensors [38]).

The results of this section are presented in Table 1, which captures the critical information from all studies in which AI methods were used to predict BG values. The table was designed to provide quick access to information about current technologies being tested. We outline the features of each study using key information, including prediction horizon $(\mathrm{PH})$ in minutes, objective population criteria, number of participants in the cohort, mean number of monitored days per patient, mean number of monitored hours per day, existence of monitoring during the overnight period, type of monitoring technology, and information about physical activity. 
Table 1. Summary of reviewed studies addressing blood glucose prediction: prediction horizon in minutes, objective population criteria, number of participants in the cohort, mean number of monitored days per patient, mean number of monitored hours per day, type of monitoring technology, existence of monitoring during the overnight period $(\mathrm{O})$ and inclusion of exercise or physical activity information $(\mathrm{E})$.

\begin{tabular}{|c|c|c|c|c|c|c|c|c|c|}
\hline Prediction horizon (min) & Population & Cohort & Days & Time & $\mathrm{O}$ & $\mathrm{E}$ & Method & Ref & Year \\
\hline $15,30,45$ & $\mathrm{~T} 1 \mathrm{D}^{\mathrm{a}}$ & 15 & 28 & $10 \mathrm{~h}$ & $\checkmark$ & & $\mathrm{ANN}^{\mathrm{b}}$ & [39] & 2010 \\
\hline 30 & T1D & 12 & 10 & $24 \mathrm{~h}$ & $\checkmark$ & & ANN & [33] & 2010 \\
\hline 75 & Critical Care & 1 & 16 & $15 \mathrm{~h}$ & $\checkmark$ & & ANN & {$[40]$} & 2010 \\
\hline 75 & T1D & 27 & 5 & $24 \mathrm{~h}$ & $\checkmark$ & & ANN & [41] & 2011 \\
\hline 30 & T1D & $5 \mathrm{VP}^{\mathrm{c}}, 1$ & 7 & $24 \mathrm{~h}$ & $\checkmark$ & $\checkmark$ & ANN & {$[42]$} & 2011 \\
\hline 30,45 & T1D & $30 \mathrm{VP}$ & 8 & $24 \mathrm{~h}$ & $\checkmark$ & & ANN & [43] & 2012 \\
\hline $15,30,60,120$ & T1D & 27 & 13 & $24 \mathrm{~h}$ & $\checkmark$ & $\checkmark$ & $\mathrm{RF}^{\mathrm{d}}$ & [44] & 2012 \\
\hline 30 & T1D & 20VP, 9 & 11,7 & $24 \mathrm{~h}$ & $\checkmark$ & & ANN & [45] & 2012 \\
\hline 30 & T1D & 10 & 3 & $24 \mathrm{~h}$ & $\checkmark$ & & $\mathrm{SVM}^{\mathrm{e}}, \mathrm{RA}^{\mathrm{f}}, \mathrm{ANN}$ & {$[46]$} & 2013 \\
\hline $15,30,45$ & T1D & 23 & 6.1 & $24 \mathrm{~h}$ & $\checkmark$ & & RA, ANN & [47] & 2013 \\
\hline $15,30,60,120$ & T1D & 27 & 13 & $24 \mathrm{~h}$ & $\checkmark$ & $\checkmark$ & SVR & {$[48]$} & 2013 \\
\hline 30 & T1D & 20 & 3 & $24 \mathrm{~h}$ & $\checkmark$ & & ANN & [49] & 2015 \\
\hline $15,30,45,60$ & T1D & 6 & 11 & $24 \mathrm{~h}$ & $\checkmark$ & $\checkmark$ & ANN & {$[50]$} & 2015 \\
\hline $30,60,120$ & $\mathrm{~T} 1 \mathrm{D}$ & 10 & 6 & $24 \mathrm{~h}$ & $\checkmark$ & $\checkmark$ & ANN & [51] & 2015 \\
\hline 30 & T1D & 15 & 13 & $24 \mathrm{~h}$ & $\checkmark$ & $\checkmark$ & ANN & {$[52]$} & 2015 \\
\hline 30 & T1D & $5 \mathrm{VP}, 1$ & 30 & $24 \mathrm{~h}$ & $\checkmark$ & & SVR & {$[53]$} & 2016 \\
\hline- & $\mathrm{T} 2 \mathrm{D}^{\mathrm{g}}$ & 346 & 1 & - & & & ANN & {$[54]$} & 2016 \\
\hline $5,15,30,45,60$ & T1D & 15 & 13 & $24 \mathrm{~h}$ & $\checkmark$ & $\checkmark$ & Kernel & {$[55]$} & 2016 \\
\hline 60 & T1D & 5 & 90 & $24 \mathrm{~h}$ & & & $\mathrm{EA}^{\mathrm{h}}$ & {$[56]$} & 2016 \\
\hline 1440 & $\mathrm{~T} 1 \mathrm{D}, \mathrm{T} 2 \mathrm{D}$ & 8 & 3 & $24 \mathrm{~h}$ & & $\checkmark$ & $\mathrm{DT}^{\mathrm{i}}$ & {$[57]$} & 2016 \\
\hline 30 & T1D & 3 & 10 & $24 \mathrm{~h}$ & $\checkmark$ & & EA & {$[58]$} & 2016 \\
\hline 30,60 & T1D & 17 & 6 & $24 \mathrm{~h}$ & $\checkmark$ & & RA & [59] & 2017 \\
\hline $60,120,150,180$ & T1D & $20 \mathrm{VP}$ & 14 & $24 \mathrm{~h}$ & $\checkmark$ & & EA & {$[60]$} & 2017 \\
\hline 0 & $\mathrm{~T} 2 \mathrm{D}$ & 3 & 23 & - & & & $\mathrm{NB}^{\mathrm{j}}$ & {$[61]$} & 2017 \\
\hline $30,60,90,120$ & T1D & 10 & 10 & $24 \mathrm{~h}$ & $\checkmark$ & & $\mathrm{KNN}^{\mathrm{k}}, \mathrm{RF}, \mathrm{EA}$ & {$[62]$} & 2017 \\
\hline 120 & T1D & $100 \mathrm{VP}$ & 14 & $24 \mathrm{~h}$ & $\checkmark$ & & EA & {$[63]$} & 2017 \\
\hline $30,60,90$ & $\mathrm{~T} 1 \mathrm{D} \& \mathrm{~T} 2 \mathrm{D}$ & 106 & $<7$ & $24 \mathrm{~h}$ & $\checkmark$ & & RA and ANN & [64] & 2017 \\
\hline
\end{tabular}

T1D: type 1 diabetes.

${ }^{\mathrm{b}} \mathrm{ANN}$ : artificial neural network.

${ }^{\mathrm{c}}$ VP: virtual patient.

${ }^{\mathrm{d}} \mathrm{RF}$ : random forest.

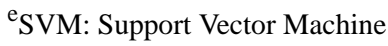

${ }^{\mathrm{R} A}$ : regression algorithm.

${ }^{\mathrm{T}}$ 2D: type 2 diabetes.

${ }^{\mathrm{h}}$ EA: evolutionary algorithm.

${ }^{\mathrm{i}} \mathrm{DT}$ : decision tree.

${ }^{\mathrm{j}} \mathrm{NB}$ : Naïve Bayes.

${ }^{\mathrm{k}} \mathrm{KNN}$ : k-nearest neighbor.

Finally, we highlight the main AI methods applied in the studies, the bibliographic reference, and the year of publication. AI for $\mathrm{BG}$ prediction has been addressed in as many as 13 parallel

lines of research. Most of these studies focused on T1D because of the inherent utility of $\mathrm{AI}$ in this condition and the availability of high-frequency data collected from patients using a CGM 
device. The results of our review reveal the range of PHs explored, from 5 to 180 minutes. Short-term predictions were the most frequently explored: 38 out of 49 studies (76\%) used PHs below 60 minutes. ANN approaches were the most widely applied methodology, but other machine learning methodologies such as RF, SVM, or RAs are being adopted with increasing frequency.

\section{Detection of Adverse Glycemic Events}

As with BG prediction, glycemic episode detection encompasses a set of tools that deal with the complexity of effective BG control. However, in this section we will not address glucose values, but instead focus on the appearance of hyperglycemic or hypoglycemic events. These tools enable us to detect the occurrence of glycemic episodes and give us the opportunity to respond promptly to their effects. In contrast to the previous section, most of the reviewed studies on this topic focus on detecting hyperglycemia or hypoglycemia in situations when it is not possible to effectively monitor BG. Therefore, most of these studies deal with real-time approaches rather than predictions of future events. We summarize the studies dealing with detection of BG excursions in Table 2. Each scenario is represented by the following features: prediction horizon in minutes, objective population criteria, number of participants in the cohort, mean number of monitored days per patient, mean number of monitored hours per day, type of monitoring technology, existence of monitoring during the overnight period, and inclusion of exercise or physical activity information. Finally, we highlight the main AI methods applied in each study, the bibliographic reference, and the year of publication. The results revealed that nine of the 14 studies (64\%) reported real-time detection systems, and 10 of these studies (93\%) were specifically focused on T1D. Table 2 shows that over six of the approaches that exclusively addressed T1D (60\%) gathered data from CGM sensors, whereas the remainder used an electroencephalogram (EEG) or self-monitoring blood glucose (SMBG) measurements. Studies focusing on T1D were performed with fewer than 15 patients, whereas studies of T2D included larger cohorts. Sensitivity and specificity were the most common outcomes used to assess the quality of approaches to glycemic detection. Although this section contains fewer papers than the one on BG prediction, we identified more than 10 research groups contributing to this topic. In particular, researchers at the Centre for Health Technologies (Faculty of Engineering and Information Technology, Sydney, Australia) have published five studies on this topic over the last 7 years.

\section{Insulin Bolus Calculators and Advisory Systems}

The most common insulin therapies for diabetics, continuous subcutaneous insulin infusion (CSII) and multiple daily insulin injections (MDI), operate according to similar principles [79]. Both utilize basal insulin (injection of long-acting basal insulin and infusion at a constant basal rate, respectively) and bolus insulin (injection of quick-acting bolus insulin and meal boluses, respectively) to cover meals or snacks. The calculation of correct insulin doses and the estimation of the amount of carbohydrates is a regular task in the daily life of many insulin-dependent patients. Bolus advisors are based on previous insulin doses, BG measurements, planned carbohydrate estimates, and other patient-specific parameters, including insulin-to-carbohydrate ratio and insulin sensitivity. Manually calculating bolus doses and counting carbohydrates can be complex and challenging because individuals must consider multiple parameters to achieve satisfactory glucose control, and miscalculation of these values may result in persistent glycemic episodes.

To support carbohydrate estimation and determination of insulin doses by patients, tools for providing bolus advice and carbohydrate estimates are increasingly being adopted. These tools seek to increase the accuracy of mealtime and correction boluses. AI has been used to provide sets of tools to improve the accuracy of carbohydrate estimates and to calculate the optimal insulin bolus for the ingested meal

We identified several studies that applied AI to systems aimed at supporting patient decisions by issuing advice regarding meals, exercise, or medication. Research groups at the Imperial College London performed an extensive study of an insulin bolus calculator based on case-based reasoning methodology [80-84]. Their approach, which manages various dynamically optimized diabetes scenarios, was proven in a clinical trial (NCT02053051) to be a safe decision support tool. Additionally, this approach was demonstrated to improve glycemic control in diabetes management when it was combined with an AP system [84]. A similar approach was presented recently by another group [85], which also proposed an insulin bolus calculator based on case-based reasoning but, in contrast to other bolus calculators, it used a novel temporal retrieval algorithm. The Center for Biomedical Engineering Research at the University of Bern performed several important and extensive studies [86-90] investigating the GoCARB system, which provides dietary advice to diabetic patients based on automatic carbohydrate counting. Their approach is based on the use of computer vision techniques, such as feature extraction and SVM, and pilot studies show it to be an excellent assistive tool. We have also found several studies that validated their approach using the UVA/Padova patient simulator. Srinivasan et al proposed the use of a set of insulin delivery profiles optimized by a PSO to find the optimal open- and closed-loop profiles for various meal compositions [91]. More recently, another study [92] presented an approach based on ANN to optimize bolus calculation by patients using CGM. The results revealed that it was better at reducing the blood glucose risk index value than other approaches. Finally, Lee et al proposed an advisory treatment system that provides insulin, meal, and exercise recommendations [93]. Their study, which compared rule-based reasoning and k-nearest neighbor algorithms, concluded that the k-nearest neighbor algorithm was best suited to this approach.

\section{Risk and Patient Stratification}

Most commercially available tools and protocols for managing diabetes are based on general models of the diabetic population or involve subsets of patients defined by simple clusterization features and easily identifiable characteristics. However, the daily lives of diabetic patients are determined by a wide range of management scenarios that are not represented in these general models. Insulin-dependent patients must manage a highly complex process to maintain suitable levels of BG. 
Table 2. Summary of reviewed studies addressing detection of adverse glycemic events: prediction horizon (PH) in minutes, objective population criteria, number of participants in the cohort, mean number of monitored days per patient, mean number of monitored hours per day, type of monitoring technology, existence of monitoring during the overnight period $(\mathrm{O})$, and inclusion of exercise or physical activity information (E),

\begin{tabular}{|c|c|c|c|c|c|c|c|c|c|c|}
\hline $\mathrm{PH}(\min )$ & Population & Cohort & Days & Time & Measurements & $\mathrm{O}$ & $\mathrm{E}$ & Method & $\operatorname{Ref}^{\mathrm{a}}$ & Year \\
\hline $0 \min$ & $\mathrm{T} 1 \mathrm{D}^{\mathrm{b}}$ & 6 & 1 day & $10 \mathrm{~h}$ & $\mathrm{EEG}^{\mathrm{c}}$ & $\checkmark$ & & $\mathrm{ANN}^{\mathrm{d}}$ & [65] & 2010 \\
\hline $0 \mathrm{~min}$ & T1D & 30 & 80-247 days & - & $\mathrm{SMBG}^{\mathrm{e}}$ & & $\checkmark$ & $\mathrm{RF}^{\mathrm{f}}, \mathrm{SVM}^{\mathrm{g}}$ & [66] & 2012 \\
\hline $0 \mathrm{~min}$ & T1D & 15 & 1 day & $10 \mathrm{~h}$ & $\mathrm{CGM}^{\mathrm{h}}$ & $\checkmark$ & & ANN, PSO $^{\mathrm{i}}$ & [67] & 2012 \\
\hline $0 \mathrm{~min}$ & T1D & 10 & 30 days & $4 \mathrm{~h}$ & CGM & & & SVM & {$[68]$} & 2013 \\
\hline $30,60 \mathrm{~min}$ & T1D & 15 & 12.5 days & $24 \mathrm{~h}$ & CGM & $\checkmark$ & $\checkmark$ & SVM & [69] & 2013 \\
\hline $0 \mathrm{~min}$ & T1D & 10 & 4.5 days & $6 \mathrm{~h}$ & CGM & & & SVM & [70] & 2013 \\
\hline $30 \mathrm{~min}$ & T1D & 10 & 17.3 days & $24 \mathrm{~h}$ & CGM & $\checkmark$ & & $\mathrm{DT}^{\mathrm{j}}$ & [71] & 2013 \\
\hline $24 \mathrm{~h}$ & $\mathrm{~T} 2 \mathrm{D}^{\mathrm{k}}$ & 163 & -1 & -1 & SMBG & & & $\mathrm{RF}$ & [72] & 2015 \\
\hline $0 \mathrm{~min}$ & T1D & 15 & 1 day & $4 \mathrm{~h}$ & CGM & & & ANN & [73] & 2014 \\
\hline $0 \mathrm{~min}$ & T1D & 10 & $-^{\mathrm{m}}$ & - $^{\mathrm{m}}$ & SMBG, ECG & $\checkmark$ & & ANN & [74] & 2014 \\
\hline $2,7,30,61-90$ days & $\mathrm{T} 1 \mathrm{D}, \mathrm{T} 2 \mathrm{D}$ & 201,323 & $-^{\mathrm{n}}$ & $-^{\mathrm{n}}$ & SMBG & & & Pattern recognition & {$[75]$} & 2014 \\
\hline $0 \mathrm{~min}$ & T1D & 15 & 1 day & $10 \mathrm{~h}$ & ECG & $\checkmark$ & $\checkmark$ & ANN & [76] & 2016 \\
\hline Past events & $\mathrm{T} 2 \mathrm{D}$ & 119695 & $>12$ days & - & $\mathrm{EHR}^{\mathrm{o}}$ & & & $N L P^{p}$ & [77] & 2016 \\
\hline $0 \mathrm{~min}$ & $\mathrm{~T} 1 \mathrm{D}, \mathrm{T} 2 \mathrm{D}$ & 500 & 1 day & $2 \mathrm{~h}$ & SMBG & & & DT, ANN & [78] & 2017 \\
\hline
\end{tabular}

${ }^{\mathrm{a}}$ Ref: reference.

$\mathrm{b}_{\mathrm{T}}$ 1D: type 1 diabetes.

${ }^{\mathrm{c}}$ EEG: electroencephalogram.

$\mathrm{d}_{\mathrm{ANN}}$ : artificial neural network.

${ }^{\text {e }}$ SMBG: self-monitoring blood glucose.

${ }^{\mathrm{f} F}$ : random forest.

gSVM: support vector machine.

${ }^{\mathrm{h}}$ CGM: continuous glucose monitoring.

${ }^{\mathrm{i}}$ PSO: particle swarm optimization.

${ }^{\mathrm{j}} \mathrm{DT}$ : decision tree.

$\mathrm{k}_{\mathrm{T}}$ 2D: type 2 diabetes.

$1_{344}$ data points.

$\mathrm{m}_{18}$ data points.

$\mathrm{n}_{787}$ data points.

${ }^{\circ}$ EHR: electronic health record.

$\mathrm{p}_{\mathrm{NLP}}$ : natural language processing.

Treatment of diabetes is governed by diverse factors, implying high intra- and interpatient variability [94]. Exercise, nutrition disturbances, age, and cardiovascular complications are just some of the long list of factors that can dramatically impact quality of life and undermine medication adherence even when patients follow their treatment regimen strictly. Such patient variability severely limits the use of general models, which cannot capture the specific physiological behaviors of individuals. Thus, an important step toward better risk detection and intervention is personalization of the system. Over the past decade, major research efforts have been devoted to developing management tools capable of stratifying patients in different segments of the population. Risk assessment and patient stratification methods are important to improving the management of diabetes, and therefore the overall health outcomes of diabetic patients, and consequently have attracted a greater share of attention from the medical community.

This category gathers all reviewed papers that systematically identified individual patients and their risk factors to manage and coordinate their care based on specific conditions and on evidence-based guidelines. Table 3 outlines the type of stratification together with the specific challenge. Main characteristics, such as number of years, cohort, and objective population, are also included. Finally, the table defines the AI methodology applied, bibliographic reference, and year of publication. 
Table 3. Summary of studies addressing risk and patient stratification.

\begin{tabular}{|c|c|c|c|c|c|c|c|}
\hline Stratification & Challenge & Period & Cohort & Population & Methods & Year & $\operatorname{Ref}^{\mathrm{a}}$ \\
\hline Complications & Group risks of retinopathy & 5 years & 55 & $\mathrm{~T}_{1} \mathrm{D}^{\mathrm{b}}$ & $\mathrm{DT}^{\mathrm{c}}, \mathrm{ANN}^{\mathrm{d}}$ & 2010 & [95] \\
\hline Disease complexity & $\begin{array}{l}\text { Group combinations of comorbid con- } \\
\text { ditions }\end{array}$ & 2 years & 15480 & Chronic diseases & Hierarchical clustering & 2011 & {$[96]$} \\
\hline Disease complexity & Group management profiles & 3 months & 239 & T1D & Hierarchical clustering & 2011 & [97] \\
\hline Disease complexity & Group management profiles & 3.5 months & 70 & $\mathrm{~T} 2 \mathrm{D}^{\mathrm{e}}$ & K-means & 2012 & {$[98]$} \\
\hline Complications & Group biomechanical foot profiles & 6 months & 97 & T1D, T2D & K-means & 2013 & [99] \\
\hline Disease complexity & Group by drug purchases & 7 years & 953 & $\mathrm{~T} 2 \mathrm{D}$ & Knowledge discovery & 2015 & [100] \\
\hline Complications & Group risks of renal disease & 3 years & 109 & $\mathrm{~T} 2 \mathrm{D}$ & K-means & 2015 & [101] \\
\hline Complications & Group risks of complications & 10 years & 1441 & T1D & Learning models & 2015 & [102] \\
\hline Complications & Group risks of complications & 5 years & 84 & T1D, T2D & $\mathrm{RA}^{\mathrm{f}}$ and $\mathrm{ANN}$ & 2015 & [103] \\
\hline Complications & Group risks of retinopathy & $<1$ year & 345 & $\mathrm{~T} 2 \mathrm{D}$ & $\mathrm{SVM}^{\mathrm{g}}, \mathrm{RF}^{\mathrm{h}}, \mathrm{DT}, \mathrm{NB}^{\mathrm{i}}$ & 2015 & [104] \\
\hline Disease complexity & Groups of blood glucose profiles & 4 months & 10 & T1D & Hierarchical clustering & 2016 & [105] \\
\hline Complications & Group personal networks types & 5 years & 1862 & $\mathrm{~T} 2 \mathrm{D}$ & RA, K-means; & 2016 & [106] \\
\hline Disease progression & Group risks of T2D progression & 5 years & 24331 & $\mathrm{~T} 2 \mathrm{D}$ & NB & 2016 & [107] \\
\hline Complications & Group risks of retinopathy & 2 years & 323378 & $\mathrm{~T} 2 \mathrm{D}$ & $\mathrm{RF}$ & 2017 & [108] \\
\hline Disease complexity & Group blood glucose profiles & 2 years & 27005 & $\mathrm{~T} 2 \mathrm{D}$ & $\mathrm{RF}$ & 2017 & [109] \\
\hline Disease complexity & Groups of HbA1c profiles & 5 years & 684 & $\mathrm{~T} 2 \mathrm{D}$ & RAs, KNN & 2017 & [110] \\
\hline Weight intervention & Group of BMI profiles & 31 years & 2540 & $\mathrm{~T} 2 \mathrm{D}$ & RA & 2017 & [111] \\
\hline Complications & $\begin{array}{l}\text { Group by retinopathy, neuropathy, or } \\
\text { nephropathy }\end{array}$ & $3,5,7$ years & 943 & $\mathrm{~T} 2 \mathrm{D}$ & RF, RA & 2018 & [112] \\
\hline
\end{tabular}

aref: reference.

$\mathrm{b}_{\mathrm{T}}$ 1D: type 1 diabetes.

${ }^{\mathrm{c}} \mathrm{DT}$ : decision tree.

${ }^{\mathrm{d}} \mathrm{ANN}$ : artificial neural network.

$\mathrm{e}_{\mathrm{T}} 2 \mathrm{D}$ : type 2 diabetes.

${ }^{\mathrm{R} A} \mathrm{~A}$ : regression algorithm.

g SVM: support vector machine.

${ }^{h} \mathrm{RF}$ : random forest.

${ }^{\mathrm{i} N B}$ : Naïve Bayes.

\section{Detection of Meals, Exercise, and Faults}

Because people with both types of diabetes need support to successfully manage their disease, solutions with higher accuracy that require less user interaction are associated with higher-quality diabetes treatments. Tools or algorithms capable of early detection of critical events affecting glycemic control, such as exercise, a meal, or an infusion set failure, are critical for systematic automation of both closed-loop and open-loop systems. Insulin-dependent patients monitoring their glucose with CGM devices use BG measurements to calculate insulin infusion rates. Consequently, failure of these devices can lead to episodes of hyperglycemia or hypoglycemia. Leal et al proposed an approach using SVM to detect correct and incorrect measurements in real-time CGM [113]. They tested their system on 23 critically ill patients and obtained promising results in patients with sepsis or septic shock. The same objective was pursued in the work performed by Turksoy et al [114], who used a k-nearest neighbor algorithm for the diagnosis of faults and the data from 51 patients to validate the performance of their approach. For the detection of inaccurate measurements by glucose meters, another study [115] developed an SVM algorithm to minimize the effect of hematocrit on glucose measurement and tested their method on 400 BG samples.

Physical activity offers multiple benefits for diabetic patients, but also complicates the management of diabetes, especially in T1D patients. Some of the factors affecting BG dynamics during exercise include the intensity, duration, and type of exercise, insulin on board, and the carbohydrate absorption rate. Tools and systems focused on automated detection of exercise could improve the accuracy of treatments. Turksoy et al also proposed the use of a k-nearest neighbor classification algorithm to automatically detect exercise type and intensity in an AP system [116]. They tested their approach in 5 T1D patients and reported a sensitivity of $98.37 \%$. Similarly, Jacobs et al proposed a regression model to automatically detect physical exercise in patients carrying an accelerometer and a heart rate sensor [117]. 
The system was assessed in 13 T1D patients, yielding a sensitivity of $97.2 \%$ and a specificity of $99.5 \%$.

Meal detection is important in AP systems that do not permit manual meal announcements and as a safety system for patients who may forget to enter meal information manually. Turksoy et al have also investigated the development of a meal detection system based on analysis of CGM signals using an unscented Kalman filter and a fuzzy system to estimate the carbohydrates content [118-120]. Their approach was validated in silico with 30 T1D patients using the UVA/Padova simulator, which revealed a sensitivity of $91.3 \%$ and an error of $23.1 \%$ in carbohydrate estimation; and in vivo using data from $11 \mathrm{~T} 1 \mathrm{D}$ patients, which revealed a sensitivity of $93.5 \%$ for meals and $68.0 \%$ for snacks.

Insulin pump failure may result in prolonged hyperglycemia or diabetic ketoacidosis. Early detection of failures could minimize the associated risk. Cescon et al proposed the use of a time-varying autoregressive model to develop a patient alert system [121]. Validation with data from 9 T1D patients during 18 weeks of infusion set wear revealed that the system had $50 \%$ sensitivity and $66 \%$ specificity.

\section{Lifestyle and Daily-Life Support in Diabetes Management}

Lifestyle management is a fundamental aspect of diabetes care. Sedentary living, stress, nonadherence to medication, lack of regular medical examinations, and bad habits can lead to discontinuation of treatment for patients with diabetes. From the time of diagnosis, patients are required to optimize their lifestyles to manage complications and other comorbid conditions, with the overall goal of enhancing their own care. Current technologies and data warehouses enable solutions that model data and make quality decisions based upon them. Decision support systems (DSSs) consist of tools focused on helping patients or doctors to manage diabetes therapies. These systems usually have monitoring features that facilitate systematic recording of information about diet, physical activity, medication, glucose measurements, etc and combine it with tools to support both patients and clinicians, with the overall goal of enhancing therapeutic outcomes.

Multiple studies aimed at developing DSSs to manage diabetes have been proposed since 2010. One of the most productive approaches is the METABO project [122-127]. This project involves monitoring and advanced features including tools to prevent future excursions, dynamically optimize care pathways, extract patterns via knowledge discovery, and guide weight loss programs. The authors conducted several pilot trials, including usability tests in 36 T1D patients. The MOSAIC project $[100,110,112,128]$, another important project, is focused on the development of a DSS for T2D management, with a special focus on the risk assessment of related complications using data mining methods. Another daily-life support system has advanced tools, such as a recommender system that employs case-based reasoning and an integrated $\mathrm{BG}$ prediction tool based on evolutionary computation [129].

Recently, Everett et al presented a DSS using machine learning to promote adherence to physical activity and weight reduction
[130]. Authors validated the system with 55 patients with prediabetes. Previously, Yom-Tov et al proposed a DSS based on a RL algorithm that automatically sends messages to patients who are following a personalized plan for physical exercise [131]. The approach was validated in 27 sedentary T2D patients. Daily-life support systems using AI tools for GDM were also investigated. A weight management proposal was presented in the MediClass system [132]. The system, which is based on the application of a natural language processing (NLP) algorithm, was validated during the postpartum visits of $600 \mathrm{GDM}$ patients. Rigla et al also investigated tools for GDM patients [133]. They proposed a mobile app based on an AI-augmented telemedicine DSS as a tool for helping GDM patients. Later, they presented a platform to remotely evaluate patients using a classifier based on a clustering algorithm and a decision tree learning algorithm [134]. The system was evaluated in 90 GDM patients. The results showed a reduction in the time devoted by clinicians to patients and in face-to-face visits per patient.

Six other studies have proposed alternatives to the manual creation of patient care workflows. The studies offer support for the design and deployment of diabetes management protocols, as well as ways to continuously improve patient tracking throughout the entire process. Cleveringa et al presented a system aimed at decreasing cardiovascular risk of T2D patients by optimizing patient care workflows $[135,136]$. The authors validated their system by administering questionnaires to 3391 T2D patients. Miller et al used a machine learning approach to extract information from drug prescriptions from electronic health record (EHR) data and identify factors associated with patient care flow deviations [23]. Another DSS with care flow tools was presented in the work of Alotaibi et al [137]. This system focuses on the management of T2D patients using advanced features, such as computerized alerts and reminders. It was tested in $20 \mathrm{~T} 2 \mathrm{D}$ patients for 6 months and resulted in reduced $\mathrm{HbA}_{1 \mathrm{c}}$ levels and improved diabetes awareness. Fernandez-Llatas et al proposed using data mining methods to enable the dynamic design of care protocols but highlighted the need for mechanisms to reduce the Spaghetti Effect and make DSSs usable by experts [138]. Contreras et al developed a diabetes management system to integrate a series of AI models and tools with an engine to manage diabetes patient care flows [139]. Finally, Suh et al proposed a dynamic care flow system that applied data clustering together with rule mining techniques to prioritize required user tasks [140].

Other tools have been proposed for improving daily-life support for diabetes therapies. Four different tools have been designed to analyze online discussion forums and social networks to extract relevant information. First, Grieves et al compared multiple machine learning techniques (decision trees, SVM, bagging, and Bayes) to analyze patients' online comments with the aim of predicting patients' assessment of hospital performance [141]. Second, Valdez et al proposed using a k-means clustering analysis to identify communication patterns both on and off Facebook [142]. They validated their tool in a cohort of 700 T2D patients. Third, Chen et al proposed clustering based on repeated bisecting k-means with the goal of obtaining patient experience information, including emotional and temporal aspects of diabetes management [143]. Finally, 
Hamon et al proposed using NLP methods to extract information about patients' skill in managing diabetes [144].

Furthermore, tools have been developed to analyze clinical appointments, medication, and therapy adherence. For example, a machine learning approach to examine medication adherence thresholds and risk of hospitalization was implemented [145]. The system implemented in the study reported by Fioravanti et al promotes patient empowerment and adherence to therapy based on the automatic generation of feedback messages [146]. Greaves et al proposed a clinical DSS that issues medication interaction alerts based on clusters with similar management recommendations [147]. A fuzzy approach was also presented by Eghbali-Zarch et al to address the problem of medication selection in T2D patients [148]. Finally, Kurasawa et al proposed a machine learning algorithm to predict missed clinical appointments and help patients continue regular doctor visits [149].

\section{Discussion}

By systematically examining high-quality articles in the PubMed database, we identified a series of studies with the goal of evaluating the latest efforts of AI-enabled solutions for diabetes management. The topics we reviewed suggest that prediction and prevention are currently being revitalized and reinforced by AI applications, whereas "safety and failure detection" has been less extensively reviewed, constituting fewer than $6 \%$ of the studies we encountered. Similarly, few investigations have delved into the application of AI techniques to early detection of critical issues such as exercise, meals, infusion set failures, and so forth. Exploiting the latest AI techniques to improve the safety of both AP systems and open-loop tools has the potential to dramatically improve performance. By contrast, research on closed-loop systems, representing 31 out of 141 of the reviewed studies (22\%), has been the most productive area for AI applications. Most of these efforts addressed fuzzy techniques, but the application of other methodologies has begun to attract increasing interest. In our opinion, researchers in this field should continue to take advantage of the latest improvements in AI and to combine them with development of the AP. A considerable number of the reviewed studies, 41 out of 141 , investigated BG, either to develop models that enable accurate predictions of $\mathrm{BG}$ concentrations (27 studies) or to detect possible BG events (14 studies). Multiple studies reported accurate prediction and detection tools that promise to improve management resources for current and future therapies. These tools include bolus advisors, as well as both lifestyle and patient stratification.
Our findings show the increasing importance of AI methods for diabetes management. We think these methods will encourage further research into the use of AI methods to extract knowledge from diabetic data. In general, the most striking advances in the application of AI techniques come from data-driven methods that learn from large datasets. The ability to collect information from individual diabetic patients has led to a shift in diabetes management systems; accordingly, systems that lack access to valuable data will face substantial hurdles. Diabetes management is geared toward tailored management of therapies, at the level of smaller strata of patients or even individuals. Thus, management protocols provided to diabetic patients should be tailored to address their needs at various points during their illness. Furthermore, the availability of genetic data, such as that provided by metabolomics analysis, has also empowered the application of AI methods to personalization of diabetes management.

The increased availability of digitized health data from diabetic populations, along with the emerging applications of AI and research trends such as the AP and personalized medicine, suggests that we are moving toward a new paradigm for management of diabetes. This new outlook proposes to achieve custom delivery of diabetes care while tailoring professional practices, medical decisions, and treatments to individual patients. On the other hand, the inclusion of intelligent algorithms in decision making has ethical implications that should be addressed by physicians and scientists. The ethical risks associated with the release of personal data should also be investigated. For example, the increasingly frequent use of health apps and the potential use of tools based on AI by insurance companies could lead to discrimination or the exclusion (or both) of some citizens from health services.

A large number of studies have already been published on the application of AI to diabetes in a broad range of management domains. Our dive into PubMed demonstrates an acceleration in the pace of research on AI-powered tools designed to predict and prevent the complications associated with diabetes. Although the available technologies and methods for diabetes management are growing exponentially in terms of quantity and quality, the potential of AI to boost effective and accurate management of diabetes has already been demonstrated in both open- and closed-loop therapies. Research in this field should continue and should seek to discover the opportunities and advantages of applying AI methodologies in diabetes management that differentiate these strategies from other classical approaches.

\section{Acknowledgments}

This work was funded by the Spanish Government through grant DPI2016-78831-C2-2-R and the European Union through Fondo Europeo de Desarrollo Regional (FEDER).

\section{Conflicts of Interest}

None declared.

\section{References}


1. American Diabetes Association. Diagnosis and classification of diabetes mellitus. Diabetes Care 2010 Jan;33 Suppl 1:S62-S69 [FREE Full text] [doi: 10.2337/dc10-S062] [Medline: 20042775]

2. IDF Diabetes Atlas 8th edition Internet. 2017 URL: https://www.idf.org/e-library/epidemiology-research/diabetes-atlas/ 134-idf-diabetes-atlas-8th-edition.html [accessed 2018-05-23] [WebCite Cache ID 6zd9GKIXJ]

3. AI Heatmap: Healthcare Emerges As Hottest Area For Deals To Artificial Intelligence Startups Internet. 2016 URL: https:/ /www.cbinsights.com/research/artificial-intelligence-investment-heatmap/ [accessed 2018-05-23] [WebCite Cache ID 6zd9TF8UK]

4. Rigla M, García-Sáez G, Pons B, Hernando M. Artificial Intelligence Methodologies and Their Application to Diabetes. J Diabetes Sci Technol 2018 Mar;12(2):303-310. [doi: 10.1177/1932296817710475] [Medline: 28539087]

5. Kavakiotis I, Tsave O, Salifoglou A, Maglaveras N, Vlahavas I, Chouvarda I. Machine Learning and Data Mining Methods in Diabetes Research. Comput Struct Biotechnol J 2017;15:104-116 [FREE Full text] [doi: 10.1016/j.csbj.2016.12.005] [Medline: 28138367]

6. Shankaracharya, Odedra D, Samanta S, Vidyarthi A. Computational intelligence in early diabetes diagnosis: a review. Rev Diabet Stud 2010;7(4):252-262 [FREE Full text] [doi: 10.1900/RDS.2010.7.252] [Medline: 21713313]

7. Nilsson N. Artificial Intelligence: a new synthesis. Burlington: Morgan Kaufmann Publishers; 1998:a.

8. Chapman P, Clinton J, Kerber R, Khabaza T, Reinartz T, Shearer C, et al. CRISP-DM 1.0 Step-by-step data mining guide. 2000. URL: http://www.crisp-dm.org/CRISPWP-0800.pdf [accessed 2018-05-23] [WebCite Cache ID 6zd94Fo1n]

9. Bothe M, Dickens L, Reichel K, Tellmann A, Ellger B, Westphal M, et al. The use of reinforcement learning algorithms to meet the challenges of an artificial pancreas. Expert Rev Med Devices 2013 Sep;10(5):661-673. [doi: 10.1586/17434440.2013.827515] [Medline: 23972072]

10. Bertachi A, Ramkissoon C, Bondia J, Vehí J. Automated blood glucose control in type 1 diabetes: A review of progress and challenges. Endocrinología, Diabetes y Nutrición 2018 Mar;65(3):172-181. [doi: 10.1016/J.ENDINU.2017.10.011]

11. Nimri R, Atlas E, Ajzensztejn M, Miller S, Oron T, Phillip M. Feasibility study of automated overnight closed-loop glucose control under MD-logic artificial pancreas in patients with type 1 diabetes: the DREAM Project. Diabetes Technol Ther 2012 Aug;14(8):728-735. [doi: 10.1089/dia.2012.0004] [Medline: 22853723]

12. Atlas E, Nimri R, Miller S, Grunberg E, Phillip M. MD-logic artificial pancreas system: a pilot study in adults with type 1 diabetes. Diabetes Care 2010 May;33(5):1072-1076 [FREE Full text] [doi: 10.2337/dc09-1830] [Medline: 20150292]

13. Nimri R, Danne T, Kordonouri O, Atlas E, Bratina N, Biester T, et al. The "Glucositter" overnight automated closed loop system for type 1 diabetes: a randomized crossover trial. Pediatr Diabetes 2013 May;14(3):159-167. [doi: 10.1111/pedi.12025] [Medline: 23448393]

14. Phillip M, Battelino T, Atlas E, Kordonouri O, Bratina N, Miller S, et al. Nocturnal glucose control with an artificial pancreas at a diabetes camp. N Engl J Med 2013 Feb 28;368(9):824-833. [doi: 10.1056/NEJMoa1206881] [Medline: 23445093]

15. Nimri R, Muller I, Atlas E, Miller S, Kordonouri O, Bratina N, et al. Night glucose control with MD-Logic artificial pancreas in home setting: a single blind, randomized crossover trial-interim analysis. Pediatr Diabetes 2014 Mar;15(2):91-99. [doi: 10.1111/pedi.12071] [Medline: 23944875]

16. Clinical trial. 2018. A Crossover Study Comparing Two Automated Insulin Delivery System Algorithms in Adolescents and Young Adults With Type 1 Diabetes URL: https://clinicaltrials.gov/ct2/show/NCT03040414 [accessed 2018-05-23] [WebCite Cache ID 6zd69AU6T]

17. Mauseth R, Wang Y, Dassau E, Kircher R, Matheson D, Zisser H, et al. Proposed clinical application for tuning fuzzy logic controller of artificial pancreas utilizing a personalization factor. J Diabetes Sci Technol 2010 Jul 01;4(4):913-922 [FREE Full text] [doi: 10.1177/193229681000400422] [Medline: 20663457]

18. Mauseth R, Hirsch I, Bollyky J, Kircher R, Matheson D, Sanda S, et al. Use of a "fuzzy logic" controller in a closed-loop artificial pancreas. Diabetes Technol Ther 2013 Aug;15(8):628-633. [doi: 10.1089/dia.2013.0036] [Medline: 23829285]

19. Mauseth R, Lord S, Hirsch I, Kircher R, Matheson D, Greenbaum C. Stress Testing of an Artificial Pancreas System With Pizza and Exercise Leads to Improvements in the System's Fuzzy Logic Controller. J Diabetes Sci Technol 2015 Sep 14;9(6):1253-1259 [FREE Full text] [doi: 10.1177/1932296815602098] [Medline: 26370244]

20. Yadav J, Rani A, Singh V. Performance Analysis of Fuzzy-PID Controller for Blood Glucose Regulation in Type-1 Diabetic Patients. J Med Syst 2016 Dec;40(12):254. [doi: 10.1007/s10916-016-0602-6] [Medline: 27714563]

21. Khooban M, Nazari Maryam Abadi D, Alfi A, Siahi M. Swarm optimization tuned Mamdani fuzzy controller for diabetes delayed model. Turk J Elec Eng \& Comp Sci 2013;21:2110-2126. [doi: 10.3906/elk-1202-21]

22. Fereydouneyan F, Zare A, Mehrshad N. Using a fuzzy controller optimized by a genetic algorithm to regulate blood glucose level in type 1 diabetes. J Med Eng Technol 2011 Jul;35(5):224-230. [doi: 10.3109/03091902.2011.569050] [Medline: $\underline{21557700]}$

23. Miller S, Nimri R, Atlas E, Grunberg EA, Phillip M. Automatic learning algorithm for the MD-logic artificial pancreas system. Diabetes Technol Ther 2011 Oct;13(10):983-990. [doi: 10.1089/dia.2010.0216] [Medline: 21774690]

24. Dinani S, Zekri M, Kamali M. Regulation of Blood Glucose Concentration in Type 1 Diabetics Using Single Order Sliding Mode Control Combined with Fuzzy On-line Tunable Gain, a Simulation Study. J Med Signals Sens 2015;5(3):131-140 [FREE Full text] [Medline: 26284169] 
25. Bothe M, Dickens L, Reichel K, Tellmann A, Ellger B, Westphal M, et al. The use of reinforcement learning algorithms to meet the challenges of an artificial pancreas. Expert Rev Med Devices 2013 Sep;10(5):661-673. [doi: 10.1586/17434440.2013.827515] [Medline: 23972072]

26. Daskalaki E, Diem P, Mougiakakou S. An Actor-Critic based controller for glucose regulation in type 1 diabetes. Comput Methods Programs Biomed 2013 Feb;109(2):116-125. [doi: 10.1016/j.cmpb.2012.03.002] [Medline: 22502983]

27. Daskalaki E, Diem P, Mougiakakou S. Model-Free Machine Learning in Biomedicine: Feasibility Study in Type 1 Diabetes. PLoS One 2016;11(7):e0158722 [FREE Full text] [doi: 10.1371/journal.pone.0158722] [Medline: 27441367]

28. Ewings S, Sahu S, Valletta J, Byrne C, Chipperfield A. A Bayesian network for modelling blood glucose concentration and exercise in type 1 diabetes. Stat Methods Med Res 2015 Jun;24(3):342-372. [doi: 10.1177/0962280214520732] [Medline: 24492795]

29. Catalogna M, Cohen E, Fishman S, Halpern Z, Nevo U, Ben-Jacob E. Artificial neural networks based controller for glucose monitoring during clamp test. PLoS One 2012;7(8):e44587 [FREE Full text] [doi: 10.1371/journal.pone.0044587] [Medline: 22952998]

30. Tang F, Wang Y. Economic Model Predictive Control of Bihormonal Artificial Pancreas System Based on Switching Control and Dynamic R-parameter. J Diabetes Sci Technol 2017 Nov;11(6):1112-1123. [doi: 10.1177/1932296817721519] [Medline: 28728434]

31. Greenwood NJC, Gunton JE. A computational proof of concept of a machine-intelligent artificial pancreas using Lyapunov stability and differential game theory. J Diabetes Sci Technol 2014 Jul;8(4):791-806 [FREE Full text] [doi: 10.1177/1932296814536271] [Medline: 25562888]

32. Narendra KS, Parthasarathy K. Identification and control of dynamical systems using neural networks. IEEE Trans Neural Netw 1990;1(1):4-27. [doi: 10.1109/72.80202] [Medline: 18282820]

33. Mougiakakou S, Bartsocas C, Bozas E, Chaniotakis N, Iliopoulou D, Kouris I, et al. SMARTDIAB: a communication and information technology approach for the intelligent monitoring, management and follow-up of type 1 diabetes patients. IEEE Trans Inf Technol Biomed 2010 May;14(3):622-633. [doi: 10.1109/TITB.2009.2039711] [Medline: 20123578]

34. Zarkogianni K, Vazeou A, Mougiakakou S, Prountzou A, Nikita K. An insulin infusion advisory system based on autotuning nonlinear model-predictive control. IEEE Trans Biomed Eng 2011 Sep;58(9):2467-2477. [doi: 10.1109/TBME.2011.2157823] [Medline: 21622071]

35. González-Olvera MA, Gallardo-Hernández AG, Tang Y, Revilla-Monsalve M, Islas-Andrade S. A discrete-time recurrent neurofuzzy network for black-box modeling of insulin dynamics in diabetic type-1 patients. Int J Neural Syst 2010 Apr;20(2):149-158. [doi: 10.1142/S0129065710002322] [Medline: 20411597]

36. Alanis A, Leon B, Sanchez E, Ruiz-Velazquez E. Blood glucose level neural model for type 1 diabetes mellitus patients. Int J Neural Syst 2011 Dec;21(6):491-504. [doi: 10.1142/S0129065711003000] [Medline: 22131301]

37. Fernandez DCJ, Gonzalez-Perez S, Ramos-Diaz JC. Artificial neural networks for closed loop control of in silico and ad hoc type 1 diabetes. Comput Methods Programs Biomed 2012 Apr;106(1):55-66. [doi: 10.1016/j.cmpb.2011.11.006] [Medline: 22178070]

38. Bailey TS, Chang A, Christiansen M. Clinical accuracy of a continuous glucose monitoring system with an advanced algorithm. J Diabetes Sci Technol 2015 Mar;9(2):209-214 [FREE Full text] [doi: 10.1177/1932296814559746] [Medline: 25370149]

39. Pérez-Gandía C, Facchinetti A, Sparacino G, Cobelli C, Gómez EJ, Rigla M, et al. Artificial neural network algorithm for online glucose prediction from continuous glucose monitoring. Diabetes Technol Ther 2010 Jan;12(1):81-88. [doi: 10.1089/dia.2009.0076] [Medline: 20082589]

40. Pappada S, Borst M, Cameron B, Bourey R, Lather J, Shipp D, et al. Development of a neural network model for predicting glucose levels in a surgical critical care setting. Patient Saf Surg 2010 Sep 09;4(1):15 [FREE Full text] [doi: 10.1186/1754-9493-4-15] [Medline: 20828400]

41. Pappada S, Cameron B, Rosman P, Bourey R, Papadimos T, Olorunto W, et al. Neural network-based real-time prediction of glucose in patients with insulin-dependent diabetes. Diabetes Technol Ther 2011 Feb;13(2):135-141. [doi: 10.1089/dia.2010.0104] [Medline: 21284480]

42. Zecchin C, Facchinetti A, Sparacino G, De Nicolao G, Cobelli C. A new neural network approach for short-term glucose prediction using continuous glucose monitoring time-series and meal information. In: Conf Proc IEEE Eng Med Biol Soc. 2011 Presented at: Annu Int Conf IEEE Eng Med Biol Soc Internet IEEE; 2011; Boston p. 5653-5656. [doi:

10.1109/IEMBS.2011.6091368]

43. Daskalaki E, Prountzou A, Diem P, Mougiakakou S. Real-time adaptive models for the personalized prediction of glycemic profile in type 1 diabetes patients. Diabetes Technol Ther 2012 Feb;14(2):168-174. [doi: 10.1089/dia.2011.0093] [Medline: 21992270]

44. Georga E, Protopappas V, Polyzos D, Fotiadis D. A predictive model of subcutaneous glucose concentration in type 1 diabetes based on Random Forests. In: Conf Proc IEEE Eng Med Biol Soc. 2012 Presented at: Annu Int Conf IEEE Eng Med Biol Soc Internet IEEE; 2012; San Diego p. 2889-2892. [doi: 10.1109/EMBC.2012.6346567] 
45. Zecchin C, Facchinetti A, Sparacino G, De NG, Cobelli C. Neural network incorporating meal information improves accuracy of short-time prediction of glucose concentration. IEEE Trans Biomed Eng 2012 Jun;59(6):1550-1560. [doi: 10.1109/TBME.2012.2188893] [Medline: 22374344]

46. Wang Y, Wu X, Mo X. A novel adaptive-weighted-average framework for blood glucose prediction. Diabetes Technol Ther 2013 Oct;15(10):792-801 [FREE Full text] [doi: 10.1089/dia.2013.0104] [Medline: 23883406]

47. Daskalaki E, Nørgaard K, Züger T, Prountzou A, Diem P, Mougiakakou S. An early warning system for hypoglycemic/hyperglycemic events based on fusion of adaptive prediction models. J Diabetes Sci Technol 2013 May 01;7(3):689-698 [FREE Full text] [doi: 10.1177/193229681300700314] [Medline: 23759402]

48. Georga EI, Protopappas VC, Ardigo D, Marina M, Zavaroni I, Polyzos D, et al. Multivariate prediction of subcutaneous glucose concentration in type 1 diabetes patients based on support vector regression. IEEE J Biomed Health Inform 2013 Jan;17(1):71-81. [doi: 10.1109/TITB.2012.2219876] [Medline: 23008265]

49. Zecchin C, Facchinetti A, Sparacino G, Cobelli C. Jump neural network for real-time prediction of glucose concentration. Methods Mol Biol 2015;1260:245-259. [doi: 10.1007/978-1-4939-2239-0_15] [Medline: 25502386]

50. Zarkogianni K, Mitsis K, Arredondo M, Fico G, Fioravanti A, Nikita K. Neuro-fuzzy based glucose prediction model for patients with Type 1 diabetes mellitus. 2014 Presented at: IEEE-EMBS Int Conf Biomed Heal Informatics; 2014; United States p. 252-255. [doi: 10.1109/BHI.2014.6864351]

51. Zarkogianni K, Mitsis K, Litsa E, Arredondo M, Fico G, Fioravanti A, et al. Comparative assessment of glucose prediction models for patients with type 1 diabetes mellitus applying sensors for glucose and physical activity monitoring. Med Biol Eng Comput 2015 Dec;53(12):1333-1343. [doi: 10.1007/s11517-015-1320-9] [Medline: 26049412]

52. Georga E, Protopappas V, Polyzos D, Fotiadis D. Online prediction of glucose concentration in type 1 diabetes using extreme learning machines. In: Conf Proc IEEE Eng Med Biol Soc. 2015 Presented at: IEEE Eng Med Biol Soc Annu Conf Internet; 2015; Milano p. 3262-3265. [doi: 10.1109/EMBC.2015.7319088]

53. Reymann M, Dorschky E, Groh B, Martindale C, Blank P, Eskofier B. Blood glucose level prediction based on support vector regression using mobile platforms. In: Conf Proc IEEE Eng Med Biol Soc. 2016 Dec Presented at: 38th Annu Int Conf IEEE Eng Med Biol Soc; 2016; Orlando p. 2990-2993. [doi: 10.1109/EMBC.2016.7591358]

54. Wang J, Wang F, Liu Y, Xu J, Lin H, Jia B, et al. Multiple Linear Regression and Artificial Neural Network to Predict Blood Glucose in Overweight Patients. Exp Clin Endocrinol Diabetes 2016 Jan;124(1):34-38. [doi: 10.1055/s-0035-1565175] [Medline: 26797861]

55. Georga E, Principe J, Polyzos D, Fotiadis D. Non-linear dynamic modeling of glucose in type 1 diabetes with kernel adaptive filters. In: Conf Proc IEEE Eng Med Biol Soc. 2016 Dec Presented at: 38th Annu Int Conf IEEE Eng Med Biol Soc; 2016 ; Orlando p. 5897-5900. [doi: 10.1109/EMBC.2016.7592070]

56. Contreras I, Vehi J. Mid-term prediction of blood glucose from continuous glucose sensors, meal information and administered insulin. : Springer; 2016 Presented at: XIV Mediterr Conf Med Biol Eng Comput - MEDICON 2016; 2016; Cyprus. [doi: 10.1007/978-3-319-32703-7_221]

57. Schindelboeck D, Praus F, Gall W. A Diabetes Self-Management Prototype in an AAL-Environment to Detect Remarkable Health States. Stud Health Technol Inform 2016;223:273-280. [Medline: 27139414]

58. Colmenar J, Winkler S, Kronberger G, Maqueda E, Botella M, Hidalgo J. Predicting Glycemia in Diabetic Patients By Evolutionary Computation and Continuous Glucose Monitoring. : ACM Press; 2016 Presented at: Proc Genet Evol Comput Conf Companion - GECCO 2016; 2016; New York p. 1393-1400. [doi: 10.1145/2908961.2931734]

59. Frandes M, Timar B, Timar R, Lungeanu D. Chaotic time series prediction for glucose dynamics in type 1 diabetes mellitus using regime-switching models. Sci Rep 2017 Jul 24;7(1):6232-6241 [FREE Full text] [doi: 10.1038/s41598-017-06478-4] [Medline: 28740090]

60. Contreras I, Vehi J, Visentin R, Vettoretti M. Towards Decision Support Systems Based upon Scenario Profile Analysis: A Hybrid Clustering Prediction for Type 1 Diabetes Aid. 2017 Presented at: 2017 IEEE/ACM Int Conf Connect Heal Appl Syst Eng Technol; 12/07/2017; Philadelphia. [doi: 10.1109/CHASE.2017.61]

61. Albers D, Levine M, Gluckman B, Ginsberg H, Hripcsak G, Mamykina L. Personalized glucose forecasting for type 2 diabetes using data assimilation. PLoS Comput Biol 2017 Apr;13(4):e1005232 [FREE Full text] [doi: 10.1371/journal.pcbi.1005232] [Medline: 28448498]

62. Hidalgo J, Colmenar J, Kronberger G, Winkler S, Garnica O, Lanchares J. Data Based Prediction of Blood Glucose Concentrations Using Evolutionary Methods. J Med Syst 2017 Aug 08;41(9):142. [doi: 10.1007/s10916-017-0788-2] [Medline: 28791547]

63. Contreras I, Oviedo S, Vettoretti M, Visentin R, Vehí J. Personalized blood glucose prediction: A hybrid approach using grammatical evolution and physiological models. PLoS One 2017;12(11):e0187754 [FREE Full text] [doi: 10.1371/journal.pone.0187754] [Medline: 29112978]

64. Fiorini S, Martini C, Malpassi D, Cordera R, Maggi D, Verri A, et al. Data-driven strategies for robust forecast of continuous glucose monitoring time-series. In: Conf Proc IEEE Eng Med Biol Soc. 2017 Dec Presented at: Proc Annu Int Conf IEEE Eng Med Biol Soc EMBS; 11-15/07/2017; Jeju p. 1680-1683. [doi: 10.1109/EMBC.2017.8037164]

65. Nguyen H, Jones T. Detection of nocturnal hypoglycemic episodes using EEG signals. 2016 Nov Presented at: Annu Int Conf IEEE Eng Med Biol; 2010; Buenos Aires p. 4930-4933. [doi: 10.1109/IEMBS.2010.5627233] 
66. Skrøvseth SO, Årsand E, Godtliebsen F, Hartvigsen G. Mobile phone-based pattern recognition and data analysis for patients with type 1 diabetes. Diabetes Technol Ther 2012 Dec;14(12):1098-1104 [FREE Full text] [doi: 10.1089/dia.2012.0160] [Medline: 23035775]

67. San PP, Ling SH, Nguyen H. Intelligent detection of hypoglycemic episodes in children with type 1 diabetes using adaptive neural-fuzzy inference system. Conf Proc IEEE Eng Med Biol Soc 2012;2012:6325-6328. [doi: 10.1109/EMBC.2012.6347440] [Medline: 23367375]

68. Jensen M, Christensen T, Tarnow L, Mahmoudi Z, Johansen M, Hejlesen O. Professional continuous glucose monitoring in subjects with type 1 diabetes: retrospective hypoglycemia detection. J Diabetes Sci Technol 2013 Jan 01;7(1):135-143. [doi: 10.1177/193229681300700116] [Medline: 23439169]

69. Georga E, Protopappas V, Ardigò D, Polyzos D, Fotiadis D. A glucose model based on support vector regression for the prediction of hypoglycemic events under free-living conditions. Diabetes Technol Ther 2013 Aug;15(8):634-643. [doi: 10.1089/dia.2012.0285] [Medline: 23848178]

70. Jensen M, Christensen T, Tarnow L, Seto E, Dencker JM, Hejlesen O. Real-time hypoglycemia detection from continuous glucose monitoring data of subjects with type 1 diabetes. Diabetes Technol Ther 2013 Jul;15(7):538-543. [doi: 10.1089/dia.2013.0069] [Medline: 23631608]

71. Eljil K, Qadah G, Pasquier M. Predicting hypoglycemia in diabetic patients using data mining techniques. 2013 Presented at: 9th Int Conf Innov Inf Technol Internet IEEE; 2013; Abu Dhabi p. 538-543. [doi: 10.1109/Innovations.2013.6544406]

72. Sudharsan B, Peeples M, Shomali M. Hypoglycemia prediction using machine learning models for patients with type 2 diabetes. J Diabetes Sci Technol 2015 Jan;9(1):86-90 [FREE Full text] [doi: 10.1177/1932296814554260] [Medline: 25316712]

73. San P, Ling S, Nuryani, Nguyen H. Evolvable rough-block-based neural network and its biomedical application to hypoglycemia detection system. IEEE Trans Cybern 2014 Aug;44(8):1338-1349. [doi: 10.1109/TCYB.2013.2283296] [Medline: 24122616]

74. Otto E, Tannan V. Evaluation of the utility of a glycemic pattern identification system. J Diabetes Sci Technol 2014 Jul;8(4):830-838 [FREE Full text] [doi: 10.1177/1932296814532210] [Medline: 24876425]

75. Nguyen LL, Su S, Nguyen H. Neural network approach for non-invasive detection of hyperglycemia using electrocardiographic signals. Conf Proc IEEE Eng Med Biol Soc 2014;2014:4475-4478. [doi: 10.1109/EMBC.2014.6944617] [Medline: 25570985]

76. Phyo PS, Sai HL, Nguyen H. Deep learning framework for detection of hypoglycemic episodes in children with type 1 diabetes. Conf Proc IEEE Eng Med Biol Soc 2016 Dec;2016:3503-3506. [doi: 10.1109/EMBC.2016.7591483] [Medline: 28269053]

77. Nunes A, Yang J, Radican L, Engel S, Kurtyka K, Tunceli K, et al. Assessing occurrence of hypoglycemia and its severity from electronic health records of patients with type 2 diabetes mellitus. Diabetes Res Clin Pract 2016 Nov;121:192-203. [doi: 10.1016/j.diabres.2016.09.012] [Medline: 27744128]

78. Zhang Y, Zhu J, Liang Y, Chen H, Yin S, Chen Z. Non-invasive blood glucose detection system based on conservation of energy method. Physiol Meas 2017 Feb;38(2):325-342. [doi: 10.1088/1361-6579/aa50cf] [Medline: 28107204]

79. Walsh J, Roberts R. Pumping insulin: verything you need to succeed on an insulin pump Internet. San Diego: Torrey Pines Pr; 2010:1-288.

80. Pesl P, Herrero P, Reddy M, Xenou M, Oliver N, Johnston D, et al. An Advanced Bolus Calculator for Type 1 Diabetes: System Architecture and Usability Results. IEEE J Biomed Health Inform 2016 Jan;20(1):11-17. [doi:

10.1109/JBHI.2015.2464088] [Medline: 26259202]

81. Herrero P, Pesl P, Reddy M, Oliver N, Georgiou P, Toumazou C. Advanced Insulin Bolus Advisor Based on Run-To-Run Control and Case-Based Reasoning. IEEE J Biomed Health Inform 2015 May;19(3):1087-1096. [doi: 10.1109/JBHI.2014.2331896] [Medline: 24956470]

82. Reddy M, Pesl P, Xenou M, Toumazou C, Johnston D, Georgiou P, et al. Clinical Safety and Feasibility of the Advanced Bolus Calculator for Type 1 Diabetes Based on Case-Based Reasoning: A 6-Week Nonrandomized Single-Arm Pilot Study. Diabetes Technol Ther 2016 Dec;18(8):487-493. [doi: 10.1089/dia.2015.0413] [Medline: 27196358]

83. Pesl P, Herrero P, Reddy M, Oliver N, Johnston D, Toumazou C, et al. Case-Based Reasoning for Insulin Bolus Advice. J Diabetes Sci Technol 2017 Dec;11(1):37-42 [FREE Full text] [doi: 10.1177/1932296816629986] [Medline: 26862136]

84. Herrero P, Bondia J, Adewuyi O, Pesl P, El-Sharkawy M, Reddy M, et al. Enhancing automatic closed-loop glucose control in type 1 diabetes with an adaptive meal bolus calculator - in silico evaluation under intra-day variability. Comput Methods Programs Biomed 2017 Jul;146:125-131. [doi: 10.1016/j.cmpb.2017.05.010] [Medline: 28688482]

85. Brown D, Aldea A, Harrison R, Martin C, Bayley I. Temporal case-based reasoning for type 1 diabetes mellitus bolus insulin decision support. Artif Intell Med 2018 Dec;85:28-42. [doi: 10.1016/j.artmed.2017.09.007] [Medline: 28986108]

86. Rhyner D, Loher H, Dehais J, Anthimopoulos M, Shevchik S, Botwey R, et al. Carbohydrate Estimation by a Mobile Phone-Based System Versus Self-Estimations of Individuals With Type 1 Diabetes Mellitus: A Comparative Study. J Med Internet Res 2016 May 11;18(5):e101 [FREE Full text] [doi: 10.2196/jmir.5567] [Medline: 27170498] 
87. Bally L, Dehais J, Nakas C, Anthimopoulos M, Laimer M, Rhyner D, et al. Carbohydrate Estimation Supported by the GoCARB System in Individuals With Type 1 Diabetes: A Randomized Prospective Pilot Study. Diabetes Care 2017 Dec;40(2):e6-e7. [doi: 10.2337/dc16-2173] [Medline: 27899490]

88. Agianniotis A, Anthimopoulos M, Daskalaki E, Drapela A, Stettler C, Diem P, et al. GoCARB in the Context of an Artificial Pancreas. J Diabetes Sci Technol 2015 May;9(3):549-555 [FREE Full text] [doi: 10.1177/1932296815583333] [Medline: 25904142]

89. Anthimopoulos M, Dehais J, Shevchik S, Ransford B, Duke D, Diem P, et al. Computer vision-based carbohydrate estimation for type 1 patients with diabetes using smartphones. J Diabetes Sci Technol 2015 May;9(3):507-515 [FREE Full text] [doi: 10.1177/1932296815580159] [Medline: 25883163]

90. Anthimopoulos MM, Gianola L, Scarnato L, Diem P, Mougiakakou SG. A food recognition system for diabetic patients based on an optimized bag-of-features model. IEEE J Biomed Health Inform 2014 Jul;18(4):1261-1271. [doi:

10.1109/JBHI.2014.2308928] [Medline: 25014934]

91. Srinivasan A, Lee JB, Dassau E, Doyle FJ. Novel insulin delivery profiles for mixed meals for sensor-augmented pump and closed-loop artificial pancreas therapy for type 1 diabetes mellitus. J Diabetes Sci Technol 2014 Sep;8(5):957-968 [FREE Full text] [doi: 10.1177/1932296814543660] [Medline: 25049364]

92. Cappon G, Vettoretti M, Marturano F, Facchinetti A, Sparacino G. A Neural-Network-Based Approach to Personalize Insulin Bolus Calculation Using Continuous Glucose Monitoring. J Diabetes Sci Technol 2018 Mar;12(2):265-272. [doi: 10.1177/1932296818759558] [Medline: 29493356]

93. Lee M, Gatton TM, Lee KK. A MonitoringAdvisory System for Diabetes Patient Management Using a Rule-Based Method and KNN. Sensors Internet 2010 Apr;10(4):3934-3953. [Medline: 22319334]

94. Bode BW. Glycemic Variability and the Role It Should Play in Diabetes Management and Blood Glucose Monitoring. US Endocrinol Internet 2008;4(2):67. [doi: 10.17925/USE.2008.04.2.67]

95. Skevofilakas M, Zarkogianni K, Karamanos B, Nikita K. A hybrid Decision Support System for the risk assessment of retinopathy development as a long term complication of Type 1 Diabetes Mellitus. Conf Proc IEEE Eng Med Biol Soc 2010;2010:6713-6716. [doi: 10.1109/IEMBS.2010.5626245] [Medline: 21096083]

96. Newcomer S, Steiner J, Bayliss E. Identifying subgroups of complex patients with cluster analysis. Am J Manag Care 2011 Aug 01;17(8):e324-e332 [FREE Full text] [Medline: 21851140]

97. Rohan J, Delamater A, Pendley J, Dolan L, Reeves G, Drotar D. Identification of self-management patterns in pediatric type 1 diabetes using cluster analysis. Pediatr Diabetes 2011 Nov;12(7):611-618. [doi: 10.1111/j.1399-5448.2010.00752.x] [Medline: 21446925]

98. Namayanja J, Janeja V. An assessment of patient behavior over time-periods: a case study of managing type 2 diabetes through blood glucose readings and insulin doses. J Med Syst 2012 Nov;36 Suppl 1:S65-S80. [doi: 10.1007/s10916-012-9894-3] [Medline: 23104240]

99. Deschamps K, Matricali G, Roosen P, Desloovere K, Bruyninckx H, Spaepen P, et al. Classification of forefoot plantar pressure distribution in persons with diabetes: a novel perspective for the mechanical management of diabetic foot? PLoS One 2013;8(11):e79924 [FREE Full text] [doi: 10.1371/journal.pone.0079924] [Medline: 24278219]

100. Sacchi L, Dagliati A, Segagni D, Leporati P, Chiovato L, Bellazzi R. Improving risk-stratification of Diabetes complications using temporal data mining. Conf Proc IEEE Eng Med Biol Soc 2015;2015:2131-2134. [doi: 10.1109/EMBC.2015.7318810] [Medline: 26736710]

101. Migliore C, Vorderstrasse A, Pan W, Melkus G. Renal Disease Risk Factors Among Risk Groups Comprised of African American Women With Type 2 Diabetes: A Secondary Analysis. Diabetes Educ 2015 Oct 22;41(5):569-581. [Medline: 26202051]

102. Lagani V, Chiarugi F, Manousos D, Verma V, Fursse J, Marias K, et al. Realization of a service for the long-term risk assessment of diabetes-related complications. J Diabetes Complications 2015 Jul;29(5):691-698. [doi: 10.1016/j.jdiacomp.2015.03.011] [Medline: 25953402]

103. Sangi M, Win K, Shirvani F, Namazi-Rad M, Shukla N. Applying a novel combination of techniques to develop a predictive model for diabetes complications. PLoS One 2015;10(4):e0121569 [FREE Full text] [doi: 10.1371/journal.pone.0121569] [Medline: 25902317]

104. Huang G, Huang K, Lee T, Weng J. An interpretable rule-based diagnostic classification of diabetic nephropathy among type 2 diabetes patients. BMC Bioinformatics 2015;16 Suppl 1:S5 [FREE Full text] [doi: 10.1186/1471-2105-16-S1-S5] [Medline: 25707942]

105. Contreras I, Quirós C, Giménez M, Conget I, Vehi J. Profiling intra-patient type I diabetes behaviors. Comput Methods Programs Biomed 2016 Nov;136:131-141. [doi: 10.1016/j.cmpb.2016.08.022] [Medline: 27686710]

106. Vassilev I, Rogers A, Kennedy A, Wensing M, Koetsenruijter J, Orlando R, et al. Social Network Type and Long-Term Condition Management Support: A Cross-Sectional Study in Six European Countries. PLoS One 2016;11(8):e0161027 [FREE Full text] [doi: 10.1371/journal.pone.0161027] [Medline: 27536988]

107. Anderson J, Parikh J, Shenfeld D, Ivanov V, Marks C, Church B, et al. Reverse Engineering and Evaluation of Prediction Models for Progression to Type 2 Diabetes: An Application of Machine Learning Using Electronic Health Records. J Diabetes Sci Technol 2015 Dec 20;10(1):6-18 [FREE Full text] [doi: 10.1177/1932296815620200] [Medline: 26685993] 
108. DuBrava S, Mardekian J, Sadosky A, Bienen EJ, Parsons B, Hopps M, et al. Using Random Forest Models to Identify Correlates of a Diabetic Peripheral Neuropathy Diagnosis from Electronic Health Record Data. Pain Med 2017 Dec 01;18(1):107-115. [doi: 10.1093/pm/pnw096] [Medline: 27252307]

109. McCoy R, Ngufor C, Van HH, Caffo B, Shah N. Trajectories of Glycemic Change in a National Cohort of Adults With Previously Controlled Type 2 Diabetes. Med Care 2017 Nov;55(11):956-964. [doi: 10.1097/MLR.0000000000000807] [Medline: 28922296]

110. Dagliati A, Malovini A, Decata P, Cogni G, Teliti M, Sacchi L, et al. Hierarchical Bayesian Logistic Regression to forecast metabolic control in type 2 DM patients. AMIA Annu Symp Proc 2016;2016:470-479 [FREE Full text] [Medline: 28269842]

111. Contreras I, Vehi J. Mid-Term Prediction of Blood Glucose from Continuous Glucose Sensors, Meal Information and Administered Insulin. XIV Mediterranean Conference on Medical and Biological Engineering and Computing 20162016 Sep 17:1137-1143. [doi: 10.1007/978-3-319-32703-7 222]

112. Dagliati A, Marini S, Sacchi L, Cogni G, Teliti M, Tibollo V, et al. Machine Learning Methods to Predict Diabetes Complications. J Diabetes Sci Technol 2017 May 12;12(2):295-302. [doi: 10.1177/1932296817706375]

113. Leal Y, Gonzalez-Abril L, Lorencio C, Bondia J, Vehi J. Detection of correct and incorrect measurements in real-time continuous glucose monitoring systems by applying a postprocessing support vector machine. IEEE Trans Biomed Eng 2013 Jul;60(7):1891-1899. [Medline: 23380841]

114. Turksoy K, Roy A, Cinar A. Real-Time Model-Based Fault Detection of Continuous Glucose Sensor Measurements. IEEE Trans Biomed Eng 2017 Dec;64(7):1437-1445. [doi: 10.1109/TBME.2016.2535412] [Medline: 26930674]

115. Shin J, Park H, Cho S, Nam H, Lee KJ. A correction method using a support vector machine to minimize hematocrit interference in blood glucose measurements. Comput Biol Med 2014 Sep;52:111-118. [doi: 10.1016/j.compbiomed.2014.06.005] [Medline: 25033022]

116. Turksoy K, Paulino T, Zaharieva D, Yavelberg L, Jamnik V, Riddell M, et al. Classification of Physical Activity: Information to Artificial Pancreas Control Systems in Real Time. J Diabetes Sci Technol 2015 Oct 06;9(6):1200-1207 [FREE Full text] [doi: 10.1177/1932296815609369] [Medline: 26443291]

117. Jacobs P, Resalat N, El YJ, Reddy R, Branigan D, Preiser N, et al. Incorporating an Exercise Detection, Grading, and Hormone Dosing Algorithm Into the Artificial Pancreas Using Accelerometry and Heart Rate. J Diabetes Sci Technol 2015 Oct 05;9(6):1175-1184 [FREE Full text] [doi: 10.1177/1932296815609371] [Medline: 26438720]

118. Samadi S, Turksoy K, Hajizadeh I, Feng J, Sevil M, Cinar A. Meal Detection and Carbohydrate Estimation Using Continuous Glucose Sensor Data. IEEE J Biomed Health Inform 2017 Dec;21(3):619-627. [doi: 10.1109/JBHI.2017.2677953] [Medline: 28278487]

119. Turksoy K, Samadi S, Feng J, Littlejohn E, Quinn L, Cinar A. Meal Detection in Patients With Type 1 Diabetes: A New Module for the Multivariable Adaptive Artificial Pancreas Control System. IEEE J Biomed Health Inform 2016 Jan;20(1):47-54 [FREE Full text] [doi: 10.1109/JBHI.2015.2446413] [Medline: 26087510]

120. Samadi S, Rashid M, Turksoy K, Feng J, Hajizadeh I, Hobbs N, et al. Automatic Detection and Estimation of Unannounced Meals for Multivariable Artificial Pancreas System. Diabetes Technol Ther 2018 Mar;20(3):235-246. [doi: 10.1089/dia.2017.0364] [Medline: 29406789]

121. Cescon M, DeSalvo D, Ly T, Maahs D, Messer L, Buckingham B, et al. Early Detection of Infusion Set Failure During Insulin Pump Therapy in Type 1 Diabetes. J Diabetes Sci Technol 2016 Dec;10(6):1268-1276 [FREE Full text] [doi: 10.1177/1932296816663962] [Medline: 27621142]

122. Fioravanti A, Fico G, Arredondo M, Leuteritz J. A mobile feedback system for integrated E-health platforms to improve self-care and compliance of diabetes mellitus patients. In: Conf Proc IEEE Eng Med Biol Soc. 2011 Presented at: Annu Int Conf IEEE Eng Med Biol Soc; 2011; Paris p. 3550-3553. [doi: 10.1109/IEMBS.2011.6090591]

123. Fico G, Fioravanti A, Arredondo M, Leuteritz J, Guillén A, Fernandez D. A user centered design approach for patient interfaces to a diabetes IT platform. Conf Proc IEEE Eng Med Biol Soc 2011;2011:1169-1172. [doi: 10.1109/IEMBS.2011.6090274] [Medline: 22254523]

124. Fico G, Arredondo M, Protopappas V, Georgia E, Fotiadis D. Mining data when technology is applied to support patients and professional on the control of chronic diseases: the experience of the METABO platform for diabetes management. Methods Mol Biol 2015;1246:191-216. [doi: 10.1007/978-1-4939-1985-7 13] [Medline: 25417088]

125. Fico G, Fioravanti A, Arredondo M, Ardigó D, Guillén A. A healthy lifestyle coaching-persuasive application for patients with type 2 diabetes. In: Conf Proc IEEE Eng Med Biol Soc. 2010 Presented at: Annu Int Conf IEEE Eng Med Biol; 2010; Paris p. 2221-2224. [doi: 10.1109/IEMBS.2010.5626185]

126. Guillen A, Colas J, Fico G, Guillen S. METABO: A new paradigm towards diabetes disease management. An innovative business model. 2011 Annu Int Conf IEEE Eng Med Biol Soc 2011:3554-3557. [doi: 10.1109/IEMBS.2011.6090592]

127. Fico G, Fioravanti A, Arredondo M, Gorman J, Diazzi C, Arcuri G, et al. Integration of Personalized Healthcare Pathways in an ICT Platform for Diabetes Managements: A Small-Scale Exploratory Study. IEEE J Biomed Health Inform 2016 Jan;20(1):29-38. [doi: 10.1109/JBHI.2014.2367863] [Medline: 25389246]

128. Dagliati A, Sacchi L, Tibollo V, Cogni G, Teliti M, Martinez-Millana A, et al. A dashboard-based system for supporting diabetes care. J Am Med Inform Assoc 2018 May 01;25(5):538-547. [doi: 10.1093/jamia/ocx159] [Medline: 29409033] 
129. Hidalgo J, Maqueda E, Risco-Martín J, Cuesta-Infante A, Colmenar J, Nobel J. glUCModel: A monitoring and modeling system for chronic diseases applied to diabetes. J Biomed Inform 2014;48:183-192. [doi: 10.1016/J.JBI.2013.12.015]

130. Everett E, Kane B, Yoo A, Dobs A, Mathioudakis N. A Novel Approach for Fully Automated, Personalized Health Coaching for Adults with Prediabetes: Pilot Clinical Trial. J Med Internet Res 2018 Feb 27;20(2):e72 [FREE Full text] [doi: 10.2196/jmir.9723] [Medline: 29487046]

131. Yom-Tov E, Feraru G, Kozdoba M, Mannor S, Tennenholtz M, Hochberg I. Encouraging Physical Activity in Patients With Diabetes: Intervention Using a Reinforcement Learning System. J Med Internet Res 2017 Oct 10;19(10):e338 [FREE Full text] [doi: 10.2196/jmir.7994] [Medline: 29017988]

132. Hazlehurst B, Lawrence J, Donahoo W, Sherwood N, Kurtz S, Xu S, et al. Automating assessment of lifestyle counseling in electronic health records. Am J Prev Med 2014 May;46(5):457-464 [FREE Full text] [doi: 10.1016/j.amepre.2014.01.001] [Medline: 24745635]

133. Rigla M, Martínez-Sarriegui I, García-Sáez G, Pons B, Hernando M. Gestational Diabetes Management Using Smart Mobile Telemedicine. J Diabetes Sci Technol 2018 Mar;12(2):260-264 [FREE Full text] [doi: 10.1177/1932296817704442] [Medline: 28420257]

134. Caballero-Ruiz E, García-Sáez G, Rigla M, Villaplana M, Pons B, Hernando M. A web-based clinical decision support system for gestational diabetes: Automatic diet prescription and detection of insulin needs. Int J Med Inform 2017 Dec;102:35-49. [doi: 10.1016/j.ijmedinf.2017.02.014] [Medline: 28495347]

135. Cleveringa F, Welsing P, van DDM, Gorter K, Niessen L, Rutten G, et al. Cost-effectiveness of the diabetes care protocol, a multifaceted computerized decision support diabetes management intervention that reduces cardiovascular risk. Diabetes Care 2010 Feb;33(2):258-263 [FREE Full text] [doi: 10.2337/dc09-1232] [Medline: 19933991]

136. Cleveringa F, Minkman M, Gorter K, van den Dock M, Rutten GEHM. Diabetes Care Protocol: effects on patient-important outcomes. A cluster randomized, non-inferiority trial in primary care. Diabet Med 2010 Apr;27(4):442-450. [doi: 10.1111/j.1464-5491.2010.02968.x] [Medline: 20536517]

137. Alotaibi MM, Istepanian R, Philip N. A mobile diabetes management and educational system for type-2 diabetics in Saudi Arabia (SAED). mHealth 2016 Aug 24;2:33-33. [doi: 10.21037/mhealth.2016.08.01] [Medline: 28293606]

138. Fernandez-Llatas C, Martinez-Millana A, Martinez-Romero A, Benedi JM, Traver V. Diabetes care related process modelling using Process Mining techniques. Lessons learned in the application of Interactive Pattern Recognition: coping with the Spaghetti Effect. Lessons learned in the application of Interactive Pattern Recognition; 2015 Presented at: Conf Proc IEEE Eng Med Biol Soc; 25-29/08/2015; Milano p. 2127-2130. [doi: 10.1109/EMBC.2015.7318809]

139. Contreras I, Kiefer S, Vehi J. Adaptive Workflows for Diabetes Management: Self-Management Assistant and Remote Treatment for Diabetes. Stud Health Technol Inform 2017;237:151-156. [Medline: 28479559]

140. Suh M, Woodbridge J, Moin T, Lan M, Alshurafa N, Samy L, et al. Dynamic Task Optimization in Remote Diabetes Monitoring Systems. Proc IEEE Int Conf Healthc Inform Imaging Syst Biol 2012 Sep;2012:3-11 [FREE Full text] [doi: 10.1109/HISB.2012.10] [Medline: 27617297]

141. Greaves F, Ramirez-Cano D, Millett C, Darzi A, Donaldson L. Use of sentiment analysis for capturing patient experience from free-text comments posted online. J Med Internet Res 2013 Nov 01;15(11):e239 [FREE Full text] [doi: 10.2196/jmir.2721] [Medline: 24184993]

142. Valdez R, Guterbock T, Fitzgibbon K, Williams I, Wellbeloved-Stone C, Bears J, et al. From loquacious to reticent: understanding patient health information communication to guide consumer health IT design. J Am Med Inform Assoc 2017 Jul 01;24(4):680-696. [doi: 10.1093/jamia/ocw155] [Medline: 28069667]

143. Chen A. Exploring online support spaces: using cluster analysis to examine breast cancer, diabetes and fibromyalgia support groups. Patient Educ Couns 2012 May;87(2):250-257. [doi: 10.1016/j.pec.2011.08.017] [Medline: 21930359]

144. Hamon T, Gagnayre R. Improving knowledge of patient skills thanks to automatic analysis of online discussions. Patient Educ Couns 2013 Aug;92(2):197-204. [doi: 10.1016/j.pec.2013.05.012] [Medline: 23769423]

145. Lo-Ciganic W, Donohue J, Thorpe J, Perera S, Thorpe C, Marcum Z, et al. Using machine learning to examine medication adherence thresholds and risk of hospitalization. Med Care 2015 Aug;53(8):720-728 [FREE Full text] [doi: 10.1097/MLR.0000000000000394] [Medline: 26147866]

146. Fioravanti A, Fico G, Salvi D, García-Betances RI, Arredondo M. Automatic messaging for improving patients engagement in diabetes management: an exploratory study. Med Biol Eng Comput 2015 Dec;53(12):1285-1294. [doi: 10.1007/s11517-014-1237-8] [Medline: 25564181]

147. Heringa M, Siderius H, Floor-Schreudering A, de Smet P, Bouvy M. Lower alert rates by clustering of related drug interaction alerts. J Am Med Inform Assoc 2017 Jan;24(1):54-59. [doi: 10.1093/jamia/ocw049] [Medline: 27107437]

148. Eghbali-Zarch M, Tavakkoli-Moghaddam R, Esfahanian F, Sepehri M, Azaron A. Pharmacological therapy selection of type 2 diabetes based on the SWARA and modified MULTIMOORA methods under a fuzzy environment. Artif Intell Med 2018 Mar 29 (forthcoming). [doi: 10.1016/j.artmed.2018.03.003] [Medline: 29606521]

149. Kurasawa H, Hayashi K, Fujino A, Takasugi K, Haga T, Waki K, et al. Machine-Learning-Based Prediction of a Missed Scheduled Clinical Appointment by Patients With Diabetes. J Diabetes Sci Technol 2016 Dec;10(3):730-736 [FREE Full text] [doi: 10.1177/1932296815614866] [Medline: 26555782] 


\section{Abbreviations}

AI: artificial intelligence

ANN: artificial neural network

AP: artificial pancreas

BG: blood glucose

CGM: continuous glucose monitoring

CSII: continuous subcutaneous insulin infusion

DSS: decision support system

DT: decision tree

DL: deep learning

EA: evolutionary algorithm

FL: fuzzy logic

GA: genetic algorithm

GDM: gestational diabetes

KDD: knowledge discovery in databases

KNN: k-nearest neighbor

MDI: multiple daily injection

NB: Naïve Bayes

NLP: natural language processing

PH: prediction horizon

RA: regression algorithm

RF: random forest

RMSE: relative mean square error

SVM: support vector machine

T1D: type 1 diabetes

T2D: type 2 diabetes

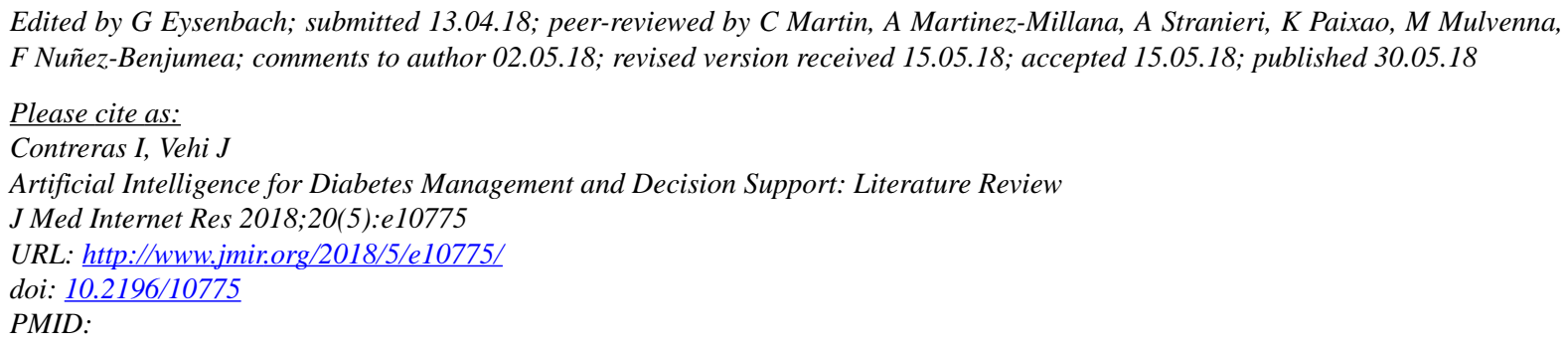

CIvan Contreras, Josep Vehi. Originally published in the Journal of Medical Internet Research (http://www.jmir.org), 30.05.2018. This is an open-access article distributed under the terms of the Creative Commons Attribution License (https://creativecommons.org/licenses/by/4.0/), which permits unrestricted use, distribution, and reproduction in any medium, provided the original work, first published in the Journal of Medical Internet Research, is properly cited. The complete bibliographic information, a link to the original publication on http://www.jmir.org/, as well as this copyright and license information must be included. 